\title{
Linear hyperbolic PDEs with noncommutative time
}

\author{
Gandalf Lechner and Rainer Verch
}

\begin{abstract}
Motivated by wave or Dirac equations on noncommutative deformations of Minkowski space, linear integro-differential equations of the form $(D+\lambda W) f=0$ are studied, where $D$ is a normal or prenormal hyperbolic differential operator on $\mathbb{R}^{n}, \lambda \in \mathbb{C}$ is a coupling constant, and $W$ is a regular integral operator with compactly supported kernel. In particular, $W$ can be non-local in time, so that a Hamiltonian formulation is not possible. It is shown that for sufficiently small $|\lambda|$, the hyperbolic character of $D$ is essentially preserved. Unique advanced/retarded fundamental solutions are constructed by means of a convergent expansion in $\lambda$, and the solution spaces are analyzed. It is shown that the acausal behavior of the solutions is well-controlled, but the Cauchy problem is ill-posed in general. Nonetheless, a scattering operator can be calculated which describes the effect of $W$ on the space of solutions to $D f=0$.

It is also described how these structures occur in the context of noncommutative Minkowski space, and how the results obtained here can be used for the analysis of classical and quantum field theories on such spaces.
\end{abstract}

Mathematics Subject Classification (2010). 46L55, 81T75; 46N50, 81T10, 58 J45.

Keywords. Linear hyperbolic PDEs, noncommutative time, noncommutative quantum field theory, quantization.

\section{Introduction}

Hyperbolic partial differential equations play a prominent role in many areas of physics, particularly in quantum field theory. They provide the dynamics for linear quantum field models which can be viewed as starting points, or building blocks, of any quantum field theory - most importantly, of quantum field models describing interactions of elementary particle physics (see e.g. [6,30,42] for a synopsis). Also in approaches to understanding the interplay of elementary particle physics and gravity [2,33,49], making use of quantum fields on curved spacetime manifolds, hyperbolic partial differential equations are of similarly prominent importance.

The mathematical questions related to such differential equations, such as theorems on the global well-posedness of the Cauchy problem and on the causal propagation character of solutions of hyperbolic partial differential operators, and 
Dirac operators, on globally hyperbolic Lorentzian manifolds, are well understood (see, for example [5]). However, the situation is quite different when considering non-commutative (or non-local) modifications of hyperbolic equations, for example

$$
\square f+w \star f=0,
$$

where $\square$ is the d'Alembertian and $\star$ a non-commutative product between $w$ and $f$, typically given by an integral expression. Similar differential equations have been studied before, even in the non-linear case [18]. However, the novel point here is that we consider the case where the product $\star$ also involves integration in the time coordinate ("non-commutative time"). Then a Hamiltonian formulation is not possible, and the usual theorems referred to above do not apply. Nonetheless, such equations appear in the context of field theories on noncommutative spacetimes; and their analysis is the topic of the present paper.

To explain our setting in more detail, it is instructive to contrast it with some well-known facts about linear hyperbolic differential equations without non-local perturbations. Consider a second order linear differential operator

$$
D: C^{\infty}\left(\mathbb{R}^{n}, \mathbb{C}^{N}\right) \rightarrow C^{\infty}\left(\mathbb{R}^{n}, \mathbb{C}^{N}\right)
$$

whose principal part is the d'Alembertian $\square=\partial^{2} / \partial x_{0}^{2}-\sum_{k=1}^{n-1} \partial^{2} / \partial x_{k}^{2} \cdot{ }^{1}$ Furthermore, let $W: C^{\infty}\left(\mathbb{R}^{n}, \mathbb{C}^{N}\right) \rightarrow C^{\infty}\left(\mathbb{R}^{n}, \mathbb{C}^{N}\right)$ be a linear map ("perturbation") and $\lambda \in \mathbb{C}$ a parameter ("coupling constant"). We are interested in the dynamics and solutions of the equation $D_{\lambda} f=0$ defined by the "perturbed" operators

$$
D_{\lambda}=D+\lambda W,
$$

and denote by $\operatorname{Sol}_{\lambda}$ the space of all solutions $f \in C^{\infty}\left(\mathbb{R}^{n}, \mathbb{C}^{N}\right)$ which have compact support in restriction to the hyperplanes of constant time $x_{0}$.

If $W$ acts as a pointwise (matrix-valued) multiplication operator, i.e. $(W f)(x)=$ $w(x) f(x)$ with $w \in C^{\infty}\left(\mathbb{R}^{n}, \mathbb{C}^{N \times N}\right)$, also $D_{\lambda}$ is a second order linear hyperbolic partial differential operator. In this case, the Cauchy problem is well-posed, i.e. to any smooth compactly supported Cauchy data, there exists a unique solution $f \in \mathrm{Sol}_{\lambda}$. Moreover, there exist uniquely determined advanced and retarded Green's operators $R_{\lambda}^{ \pm}: C_{0}^{\infty}\left(\mathbb{R}^{n}, \mathbb{C}^{N}\right) \rightarrow C^{\infty}\left(\mathbb{R}^{n}, \mathbb{C}^{N}\right)$ such that $D_{\lambda} R_{\lambda}^{ \pm} g=g=R_{\lambda}^{ \pm} D_{\lambda} g$ for all $g \in C_{0}^{\infty}\left(\mathbb{R}^{n}, \mathbb{C}^{N}\right)$ and $\operatorname{supp}\left(R_{\lambda}^{ \pm} g\right) \subset J^{ \pm}(\operatorname{supp} g)$, where $J^{ \pm}(\operatorname{supp} g)$ is the causal future $(+)$, resp. causal past $(-)$ of $\operatorname{supp} g$. Defining the causal propagator $R_{\lambda}=R_{\lambda}^{-}-R_{\lambda}^{+}$, it then holds that $R_{\lambda}$ maps $C_{0}^{\infty}\left(\mathbb{R}^{n}, \mathbb{C}^{N}\right)$ onto Sol $\lambda$, see Sec. 2 and the literature cited there for details. As an aside, we remark that this holds more generally for the case that $D$ is a second order linear operator with metric principal

\footnotetext{
${ }^{1} D$ is implicitly regarded as a second order partial differential operator on Minkowski spacetime with metric principal part, hence we use the convention to denote elements of $\mathbb{R}^{n}$ as $x=\left(x_{0}, x_{1}, \ldots, x_{n-1}\right)$ where $x_{0}$ is viewed as time-coordinate and the $x_{k}, k=1, \ldots, n-1$ are spatial coordinates.
} 
part on a globally hyperbolic Lorentzian manifold, or that $D$ is a Dirac-type operator, see [20]. Therefore, our restriction here to the case of $n$-dimensional Minkowski spacetime is mainly for the sake of simplicity.

Instead of the Cauchy problem, one can also study the scattering problem. To this end, one first introduces the Møller operators. The Møller operators and correspondingly the scattering operator are known to exist under quite general conditions on the potential $w$, a sufficient (but not necessary) condition is $w \in$ $C_{0}^{\infty}\left(\mathbb{R}^{n}, \mathbb{C}^{N \times N}\right)$. In this situation, there are $\tau_{ \pm} \in \mathbb{R}$ so that $w$ vanishes on the two future/past regions $\Sigma_{\tau_{ \pm}}^{ \pm}:=\left\{x \in \mathbb{R}^{n}: \pm x_{0}> \pm \tau_{ \pm}\right\}$. The Møller operators are then defined by

$$
\Omega_{\lambda, \pm}: \operatorname{Sol}_{\lambda} \rightarrow \operatorname{Sol}_{0}, \quad R_{\lambda} g \mapsto R_{0} g, \quad \operatorname{supp} g \subset \Sigma_{\tau_{ \pm}}^{ \pm},
$$

and the scattering operator by

$$
S_{\lambda}=\Omega_{\lambda,+}\left(\Omega_{\lambda,-}\right)^{-1}: \operatorname{Sol}_{0} \rightarrow \operatorname{Sol}_{0} .
$$

The scattering operator maps the past asymptotics of a solution of $D_{\lambda}$ to its future asymptotics, and thus provides partial information about the dynamics described by $D_{\lambda}$. We note that this way of describing the scattering process is analogous to the concept of "relative Cauchy evolution" which was studied in the context of local general covariant quantum field theory $[3,22,32]$.

To treat more general perturbations $W$, it is useful to reformulate the equation $D_{\lambda} f=0$ in a Cauchy data formulation. That is, one considers the Cauchy data $u_{\lambda, t}$ of a function at $x_{0}=t$, so that the wave equation takes the form of a one-parametric evolution equation

$$
\frac{d}{d t} u_{\lambda, t}+A_{\lambda, t} u_{\lambda, t}=0,
$$

where for any real $t, A_{\lambda, t}$ is a linear operator on the space of Cauchy data. If (1.1) is the evolution equation corresponding to a perturbation $W$ acting multiplicatively, then the $A_{\lambda, t}$ are partial differential operators. However, this is not necessary for (1.1) to have a good solution theory. In fact, the $A_{\lambda, t}$ can be fairly general pseudodifferential operators, or integro-differential operators, for example (see, e.g. [43] and literature cited therein as just one sample reference).

However, what is clearly required in this approach is that $W$ acts "locally in time" so as to allow an equivalent rewriting of the wave equation $D_{\lambda} f=0$ in terms of (1.1) where the perturbation $W$ can be re-expressed by a family of operators $A_{\lambda, t}$, each acting on the Cauchy data $u_{\lambda, t}$ of the solution $f$ at time $x_{0}=t$. There exist perturbations where $W$ is not local in time in this sense and where, hence, the perturbed wave equation $D_{\lambda} f=0$ cannot be cast into the form of an evolution equation of the type (1.1). Typical examples are integral operators involving integration over the time variable. 
It is our main aim to analyze such non-local in time hyperbolic differential equations with regard to the Cauchy and scattering problem. Our starting point is a wave operator $D$ as before, or a pre-normally hyperbolic operator $D$, see Sec. 2. Known results on the existence and uniqueness of advanced and retarded Green's operators $R^{ \pm}$for $D$ will also be summarized there. Then we consider a perturbation $W$ of $D$ which is a $C_{0}^{\infty}$-kernel operator, i.e.

$$
(W f)(x)=\int d y w(x, y) f(y)
$$

with $w \in C_{0}^{\infty}\left(\mathbb{R}^{n} \times \mathbb{R}^{n}, \mathbb{C}^{N \times N}\right)$, and as before, the perturbed operators $D_{\lambda}=$ $D+\lambda W$.

The main result of Sec. 2.2 is that, provided that $|\lambda|$ is sufficiently small, there exist fundamental solutions (Green's operators) $R_{\lambda}^{ \pm}$for $D_{\lambda}$, distinguished by the property that

$$
D_{\lambda} R_{\lambda}^{ \pm} f=f=R_{\lambda}^{ \pm} D_{\lambda} f
$$

for all $f \in C_{0}^{\infty}\left(\mathbb{R}^{n}, \mathbb{C}^{N}\right)$, and a localization property of $\operatorname{supp}\left(R_{\lambda}^{ \pm} f\right)$ which is dominated by the causal propagation of the unperturbed fundamental solutions $R^{ \pm}$. Moreover, we show that such Green's operators for $D_{\lambda}$ are unique. For Cauchy data imposed outside of the support region of $w$, i.e. outside the causal closure of some set $K$ such that supp $w \subset K \times K$, the Cauchy problem for $D_{\lambda} f=0$ turns out to be well posed. In contrast, we will also show that there are $C_{0}^{\infty}$-kernels $w$ such that the Cauchy problem for $D_{\lambda} f=0$ is ill posed; both the existence and uniqueness of its solutions can break down in this situation.

Nonetheless, we will establish that, provided $|\lambda|$ is sufficiently small, it is possible to define a scattering operator, relying on the well-posedness of $D_{\lambda} f=0$ for Cauchy data imposed outside of the support region of $w$.

These findings allow, from our point of view, to draw the following conclusion: If the perturbation $W$ is non-local in time, one can in general not expect that the resulting wave equation $D_{\lambda} f=0$ permits a well-posed Cauchy problem, or that its solutions propagate strictly causally. But for certain values of the coupling - in particular, for small $\lambda-$ the term $\lambda W$ can be considered as a small perturbation of the wave operator $D$, so that the dynamics is still mainly determined by the hyperbolic character of $D$ and therefore admits unique advanced and retarded Green's operators $R_{\lambda}^{ \pm}$. In fact, it turns out that $R_{\lambda}^{ \pm}$is, in a suitable sense, meromorphic in $\lambda$. However, when $|\lambda|$ is made large, it may happen that the hyperbolic character of $D$ is no longer the dominating contribution to the dynamics. Therefore, we think that the scattering of Cauchy data in the past of $K$ to the future of $K$, described by the scattering operator $S_{\lambda}$, which exists for sufficiently small $|\lambda|$, should actually be seen as the generalization, or replacement, of the well-posedness of the Cauchy problem for the wave equation $(D+\lambda W) f=0$ when $W$ is non-local in time. We also show that the "generator" of the scattering operator $S_{\lambda}$ with respect 
to variation of the coupling parameter $\lambda$ is given by $R W$, i.e.

$$
\left.\frac{d\left(S_{\lambda} f_{0}\right)}{d \lambda}\right|_{\lambda=0}=R W f_{0}
$$

for all $f_{0} \in \mathrm{Sol}_{0}$. While this quantity might seem a quite weak replacement for the full dynamics as described by the Cauchy problem, it is actually an important object in field theory.

This brings us to our motivations, which are related to quantum physics, and more particularly quantum field theory on noncommutative spacetimes [17,21, 28, $29,38,41,44]$. Here certain non-commutative multiplication operators, such as the Moyal product [26], play a prominent role. In Sec. 3, we consider perturbation terms given by star products, i.e. $W f=w \star f$ with a product $\star$ recalled there. Such perturbations are limits of $C_{0}^{\infty}$-kernel operators, and we discuss two examples: The Moyal product itself, and a "locally noncommutative star product" as discussed in $[14,31,34]$. Both these perturbations do not have smooth and compactly supported integral kernels (in each example, one of the two properties fails), but one always has a family $W_{\varepsilon}$ of $C_{0}^{\infty}$-kernel operators such that $W_{\varepsilon} \rightarrow W$ for $\varepsilon \rightarrow 0$. One thus obtains, for each positive $\varepsilon$, scattering operators $S_{\varepsilon, \lambda}$ provided $|\lambda|$ is sufficiently small (possibly depending on $\varepsilon$ ). Nevertheless, the "generators" $d\left(S_{\varepsilon, \lambda}\right) /\left.d \lambda\right|_{\lambda=0}=R W_{\varepsilon}$ are independent of $\lambda$, and one can argue that in the context of quantum field theory, these are mainly the objects one is interested in. For the case of Dirac operators and the Moyal product, such an investigation can also be found in the thesis [8].

In Sec. 4, we consider the quantization of (solutions of) the wave equations, or Dirac-type equations, in terms of assigning abstract CCR-algebras or CARalgebras to the corresponding solution spaces. This procedure is entirely standard; we establish that, under suitable - very general - conditions, the scattering operators $S_{\lambda}$ defined on $S_{0} l_{0}$ for underlying $C_{0}^{\infty}$-kernel operators $W$ and for sufficiently small $|\lambda|$ induce $C^{*}$-algebra morphisms $s_{\lambda}$ on the associated CCR or CAR algebras of the quantized fields. They are also called "scattering morphisms" or "scattering Bogoliubov transformations".

In the case of star products that are local in time, it was argued in [13] (see also [48]) that these morphisms provide a natural assignment of quantum field observables to an "algebra of non-commutative coordinates" seen, at least tentatively, from a perspective of Connes' spectral geometry [15].

With the analysis of non-local in time perturbations carried out in the present paper, one can expect to obtain similar results also in the case of fully noncommutative spacetime, including noncommutative time. In particular, we expect to find a link to the novel deformation technique of "warped convolution" [7, 12, 27, 28]. We therefore expect that our results will be helpful in comparing different approaches to quantum field theory on noncommutative spacetimes, and provide tools to extract the relevant physical effects. 
2. (Pre-)normally hyperbolic differential operators with smoothing compactly localized perturbations

2.1. Basic definitions and preliminaries. In this subsection, we set up our notation and introduce the objects of our investigations. Starting with the basic geometric data, Minkowski space $\mathbb{R}^{n}, n \geq 2$, is endowed with its standard metric of signature $+-\cdots-$, and we put $s:=n-1$. The causal future (+)/past (-) of a set $B \subset \mathbb{R}^{n}$ is denoted $J^{ \pm}(B)$. In particular, $V^{ \pm}:=J^{ \pm}(\{0\})$ is the forward/backward light cone. A subset $M$ of Minkowski space is called causally convex if for any causal curve in $\mathbb{R}^{n}$ with endpoints in $M$, the whole curve lies in $M$. The term Cauchy hyperplane is used to refer to Cauchy surfaces of the form $\Sigma=\Sigma_{t}=\{t\} \times \mathbb{R}^{s}$, $t \in \mathbb{R}$, and we also employ the short hand notations $\Sigma_{t}^{ \pm}$for the interior of $J^{ \pm}\left(\Sigma_{t}\right)$.

We write

$$
\mathscr{C}^{\infty}:=C^{\infty}\left(\mathbb{R}^{n}, \mathbb{C}^{N}\right), \quad \mathscr{C}_{0}^{\infty}:=C_{0}^{\infty}\left(\mathbb{R}^{n}, \mathbb{C}^{N}\right), \quad \mathscr{L}^{2}:=L^{2}\left(\mathbb{R}^{n}, \mathbb{C}^{N}\right),
$$

for the space of smooth functions, smooth functions of compact support, and (equivalence classes of) Lebesgue square integrable functions $f: \mathbb{R}^{n} \rightarrow \mathbb{C}^{N}$, respectively, where $N \in \mathbb{N}$ is some integer. For subsets $K \subset \mathbb{R}^{n}$, we write $\mathscr{C}^{\infty}(K)$ for the subspace of $\mathscr{C}^{\infty}$ of functions with support in $K$, and analogously for the other spaces. All these spaces are endowed with their standard topologies, see e.g. [46]. The scalar product in $\mathscr{L}^{2}$ is denoted $\langle\cdot, \cdot\rangle$, and the one in $\mathbb{C}^{N}$ by $(\cdot, \cdot)$, i.e. $\langle f, g\rangle=\int d x(f(x), g(x))$. The associated norms are $\|\cdot\|_{2}$ and $|\cdot|$, respectively. Sometimes integrals of the form $\int_{\Sigma_{t}} d x(f(t, x), g(t, x))$ will be abbreviated as $\int_{\Sigma_{t}}(f, g)$ for typographical reasons.

By "differential operator" we shall always mean linear finite order differential operator with smooth coefficients, and by "Cauchy data $u$ on some Cauchy hyperplane $\Sigma$ ", we shall always mean smooth compactly supported Cauchy data (sometimes also called $\mathscr{C}_{0}^{\infty}$-Cauchy data). In the context of a first order (in time) differential operator, this is a function $u \in \mathscr{C}_{0}^{\infty}(\Sigma)$, whereas in the context of a second order operator, this is a pair of functions, $u \in \mathscr{C}_{0}^{\infty}(\Sigma) \times \mathscr{C}_{0}^{\infty}(\Sigma)$. Given a differential operator $D$ or a perturbation thereof, we will write that " $f$ is a solution of $D$ " as shorthand for " $f$ is a solution to the equation $D f=0$ ".

In the following, we will study differential operators of the form $D_{\lambda}=D+\lambda \mathrm{W}$, where $D$ is a (pre-)normally hyperbolic differential operator, $\lambda \in \mathbb{C}$ a coupling constant, and $W$ some non-local perturbation term. These objects are defined next.

Definition 2.1 ((Pre-)normally hyperbolic differential operators).

a) A linear differential operator $D$ on $\mathscr{C}^{\infty}$ is called normally hyperbolic if there exist smooth matrix-valued functions $U^{0}, \ldots, U^{s}, V: \mathbb{R}^{n} \rightarrow \mathbb{C}^{N \times N}$ such 
that

$$
D=\frac{\partial^{2}}{\partial x_{0}^{2}}-\sum_{k=1}^{s} \frac{\partial^{2}}{\partial x_{k}^{2}}+\sum_{\mu=0}^{s} U^{\mu}(x) \frac{\partial}{\partial x_{\mu}}+V(x) .
$$

b) A linear differential operator $D$ on $\mathscr{C}^{\infty}$ is called prenormally hyperbolic if $D$ is of first order, i.e. $D=\sum_{\mu=0}^{s} U^{\mu}(x) \frac{\partial}{\partial x_{\mu}}+V(x)$ with smooth $U^{0}, \ldots, U^{s}$, $V: \mathbb{R}^{n} \rightarrow \mathbb{C}^{N \times N}$, and there exists another first order differential operator $D^{\prime}$ on $\mathscr{C}^{\infty}$ such that $D^{\prime} D$ is normally hyperbolic.

Whereas the first definition is standard in the context of wave equations (see for example [5,50]), the second one is taken from [35, Def. 1], and basically tailored towards a convenient description of Dirac operators. In fact, the Dirac operator $D=-i \sum_{\mu=0}^{s} \gamma^{\mu} \frac{\partial}{\partial x_{\mu}}+V(x)$ is prenormally hyperbolic when the matrices $\gamma^{\mu}$ generate an irreducible representation of the complexified Clifford algebra $\mathbb{C} l_{1, s}$ $[16,45]$, and $V: \mathbb{R}^{n} \rightarrow \mathbb{C}^{N \times N}$ is smooth. We also mention that with $D$, also $D^{\prime}$ is prenormally hyperbolic.

For our analysis, both the normally hyperbolic and the prenormally hyperbolic case are equally well suited, and we will thus consider an arbitrary (pre-)normally hyperbolic differential operator $D$.

In [35], it was shown that prenormally hyperbolic operators inherit many of the well-known properties of normally hyperbolic ones [5], see also [13, 20, 40] for corresponding arguments for Dirac operators. We summarize here the characteristic features of (pre-)normally hyperbolic operators we will rely on in this article.

Theorem 2.2 (Properties of (pre-)normally hyperbolic differential operators). Let $D$ be a (pre-)normally hyperbolic differential operator. Then

D1) $D: \mathscr{C}^{\infty} \rightarrow \mathscr{C}^{\infty}$ is a linear continuous map.

D2) $D$ is local in the sense that $\operatorname{supp}(D f) \subset \operatorname{supp} f$ for any $f \in \mathscr{C}^{\infty}$.

D3) For any Cauchy hyperplane $\Sigma$ and any Cauchy data $u$ on $\Sigma$, there exists unique $f_{0}[u] \in \mathscr{C}^{\infty}$ such that $D f_{0}[u]=0$ and $\left.f_{0}[u]\right|_{\Sigma}=u$ (for prenormally hyperbolic D), respectively $\left.f_{0}[u]\right|_{\Sigma}=u_{1},\left.\partial_{0} f_{0}[u]\right|_{\Sigma}=u_{2}, u=\left(u_{1}, u_{2}\right)$ (for normally hyperbolic $D$ ).

D4) D has unique advanced and retarded fundamental solutions, i.e. continuous linear maps $R^{ \pm}: \mathscr{C}_{0}^{\infty} \rightarrow \mathscr{C}^{\infty}$ uniquely determined by the conditions that $D R^{ \pm} f=f=R^{ \pm} D f$ and $\operatorname{supp}\left(R^{ \pm} f\right) \subset J^{ \pm}(\operatorname{supp} f)$ for all $f \in \mathscr{C}_{0}^{\infty}$.

We define the causal propagator as ${ }^{2}$

$$
R:=R^{-}-R^{+},
$$

\footnotetext{
${ }^{2}$ Also called "Green's operator". Note that the sign convention used here differs from the one in the previous article [13].
} 
and the space of all solutions with compact support on Cauchy hyperplanes as

$$
\begin{array}{r}
\operatorname{Sol}_{0}:=\left\{f \in \mathscr{C}^{\infty}: D f=0, \quad \operatorname{supp} f \subset\left(V^{+}+a_{+}\right) \cup\left(V^{-}+a_{-}\right)\right. \\
\left.\quad \text { for some } a_{ \pm} \in \mathbb{R}^{n}\right\} .
\end{array}
$$

We then furthermore have

D5) The formal adjoint of $D$ w.r.t. $\langle\cdot, \cdot\rangle$, denoted $D^{*}$, is also (pre-)normally hyperbolic and thus satisfies D1)-D4) as well. The retarded/advanced fundamental solutions of $D^{*}$, denoted $S^{ \pm}$, are related to $R^{ \pm}$by $S^{ \pm}=\left(R^{\mp}\right)^{*}$.

D6) Let $\Sigma$ be a Cauchy hyperplane and $\Sigma$ an open causally convex neighborhood of $\Sigma$. Then

$$
\mathrm{Sol}_{0}=R \mathscr{C}_{0}^{\infty}(\Sigma)
$$

D7) For any causally convex subset $M \subset \mathbb{R}^{n}$, the restriction of $D$ to $M$ satisfies properties analogous to D1)-D6). The retarded and advanced fundamental solutions $R_{M}^{ \pm}$of $\left.D\right|_{M}: \mathscr{C}^{\infty}(M) \rightarrow \mathscr{C}^{\infty}(M)$ are the restrictions (in domain and range) of the $R^{ \pm}, R_{M}^{ \pm}: \mathscr{C}_{0}^{\infty}(M) \rightarrow \mathscr{C}^{\infty}(M)$.

Items D1) and D2) hold for all linear differential operators, and the proofs of $D 3)-D 7$ ) for the normally hyperbolic case can be found in [5]. For the prenormal case, $D 3$ - $-D 4$ ) have been proven explicitly by Mühlhoff [35], and also D5)-D7) can be quickly extracted from his construction of fundamental solutions.

The properties D1)-D7) are not all independent of each other. For example, D7) can be deduced from the uniqueness of the fundamental solutions, and leads to a functorial assignment from the category of all globally hyperbolic sub-spacetimes of $\mathbb{R}^{n}$, with isometric embeddings as arrows, to the corresponding solution spaces $[3,5,22]$.

The second essential input in our analysis is a non-local perturbation term. As explained in the Introduction, we are interested in describing star product multipliers, or approximate version thereof, which suggests to consider integral operators with $\mathscr{C}_{0}^{\infty}$-kernels.

Let us now motivate this choice also from a mathematical perspective. Given a linear map $W: \mathscr{C}^{\infty} \rightarrow \mathscr{C}^{\infty}$, a smooth function $f \in \mathscr{C}^{\infty}$ can be a solution of the perturbed operator $D_{\lambda}=D+\lambda W$ only if $W f \in D \mathscr{C}^{\infty}$. In the extreme case that $W \mathscr{C}^{\infty} \cap D \mathscr{C}^{\infty}=\{0\}, f$ is a solution of $D_{\lambda}$ if and only if $D f=0$ and $W f=0$ separately. Such solutions can exist, and there are even examples where any solution of $D f=0$ automatically also satisfies $W f=0$. However, these solutions are uninteresting from our point of view, as they are just solutions of the unperturbed equation and in particular do not depend on the coupling $\lambda$. In this situation, $D$ and $W$ completely decouple, and the scattering at $W$ will be trivial. 
We will therefore rather consider situations where $W \mathscr{C}^{\infty} \subset D \mathscr{C}^{\infty}$, where an interesting solution theory for $D_{\lambda}$ is not ruled out from the beginning. As $\mathscr{C}_{0}^{\infty} \subset D \mathscr{C}^{\infty}$ by the existence of Green's operators $R^{ \pm}$postulated above, this will in particular be the case when $W \mathscr{C}^{\infty} \subset \mathscr{C}_{0}^{\infty}$. In this situation, any $f \in \mathscr{C}^{\infty}$ satisfies $D_{\lambda} f=D f+\lambda W f=D\left(f+\lambda R^{ \pm} W f\right)$, which vanishes if $\left(1+\lambda R^{+} W\right) f=R h$ for some $h \in \mathscr{C}_{0}^{\infty}$. Hence many solutions will exist if $\left(1+\lambda R^{ \pm} W\right)$ can be inverted. Formally, the inverse is given by $\left(1+\lambda R^{ \pm} W\right)^{-1}=\sum_{k=0}^{\infty}\left(-\lambda R^{ \pm} W\right)^{k}$, but convergence of this series in a useful topology is not automatic.

In the next section, we will study this question in an $\mathscr{L}^{2}$-setting, and to this end, it is necessary that $W$ is regular enough to make $R^{ \pm} W$ bounded in an $\mathscr{L}^{2}$-operator norm. These requirements can most easily be met when taking $W$ to be a $\mathscr{C}_{0}^{\infty}$-kernel operator.

Definition $2.3\left(\mathscr{C}_{0}^{\infty}\right.$-kernel operator). A $\mathscr{C}_{0}^{\infty}$-kernel operator is a mapping $W$ : $\mathscr{C}^{\infty} \rightarrow \mathscr{C}^{\infty}$ which can be represented as

$$
(W f)(x):=\int d y w(x, y) f(y), \quad f \in \mathscr{C}^{\infty},
$$

where $w \in \mathscr{C}_{0}^{\infty}\left(\mathbb{R}^{n} \times \mathbb{R}^{n}, \mathbb{C}^{N \times N}\right)$. The family of all $\mathscr{C}_{0}^{\infty}$-kernel operators will be denoted $\mathcal{W}$

The relevant properties of $\mathscr{C}_{0}^{\infty}$-kernel operators that we will use are the following.

Lemma 2.4 (Properties of $\mathscr{C}_{0}^{\infty}$-kernel operators). Let $W$ be a $\mathscr{C}_{0}^{\infty}$-kernel operator. Then

W1) There exists a compact set $K \subset \mathbb{R}^{n}$ such that $W \mathscr{C}^{\infty} \subset \mathscr{C}_{0}^{\infty}(K)$, and $W f=0$ for all $f$ with supp $f \cap K=\emptyset$,

W2) $W$ extends to a continuous linear map $W: \mathscr{L}^{2} \rightarrow \mathscr{C}_{0}^{\infty}(K)$.

W3) The adjoint $W^{*}$ of $W$ w.r.t. $\langle\cdot, \cdot\rangle$ is also a $\mathscr{C}_{0}^{\infty}$-kernel operator,

W4) For any differential operator $Q$, also $W Q$ and $Q W$ satisfy W1)-W3).

Proof. W1) is clearly satisfied for any compact $K$ such that supp $w \subset K \times K$.

$W 2$ ) For compact $B \subset \mathbb{R}^{n}$, and every $a \in \mathbb{N}_{0}$, we find by a routine estimate

$$
\sup _{\substack{x \in B \\|\alpha| \leq a}}\left|\partial_{x}^{\alpha} W_{w} f(x)\right| \leq C_{a}\|f\|_{2} .
$$

Thus $W_{w}$ extends to a continuous map $W_{w}: \mathscr{L}^{2} \rightarrow \mathscr{C}_{0}^{\infty}(K)$. Since $W_{w}{ }^{*}=W_{w^{*}}$ with $w^{*}(x, y)=w(y, x)^{*}$, the same holds for the adjoint $W_{w}{ }^{*}$, i.e. we have also shown W3). Finally, acting with a differential operator $Q$ from the left on $W$ just results in a different $\mathscr{C}_{0}^{\infty}$-kernel, as multiplication and differentiation preserve $\mathscr{C}_{0}^{\infty}$. For the action from the right, $W Q$, one has to use integration by parts to arrive at the same conclusion. This shows W4). 
The following investigations will be based on a (pre-)normally hyperbolic differential operator $D$ and a $\mathscr{C}_{0}^{\infty}$-kernel operator $W$. By $K$, we will always refer to its "support", i.e. a compact subset of $\mathbb{R}^{n}$ as in Wl). Our requirements on $W$ can probably be relaxed (see also the remarks in Section 3), in particular regarding the smoothing property $W 2$ ). However, for the sake of simplicity, we stick to $\mathscr{C}_{0}^{\infty}$-kernel operators for now.

2.2. Fundamental solutions and the Cauchy problem. Having fixed a (pre-) normally hyperbolic $D$ and a $\mathscr{C}_{0}^{\infty}$-kernel operator $W \in \mathcal{W}$, we now consider, $\lambda \in \mathbb{C}$

$$
D_{\lambda}:=D+\lambda W
$$

which is defined as a continuous linear map $\mathscr{C}^{\infty} \rightarrow \mathscr{C}^{\infty}$. The first main step in our investigation will be the construction of advanced and retarded fundamental solutions for $D_{\lambda}$ (for small enough $|\lambda|$ ). These fundamental solutions will first be constructed in a suitable neighborhood of the support $K$ of the perturbation, and then on all of $\mathbb{R}^{n}$.

To introduce this neighborhood, pick two numbers $\tau_{-}<\tau_{+}$such that the time slice

$$
M_{\tau}:=\left\{x: \tau_{-}<x_{0}<\tau_{+}\right\}=\Sigma_{\tau_{-}}^{+} \cap \Sigma_{\tau_{+}}^{-}
$$

contains $K$, where $x=\left(x_{0}, \ldots, x_{n-1}\right)$ are the standard Cartesian coordinates of $\mathbb{R}^{n}$. This notation will be used throughout the article.

Restrictions to $M_{\tau}$ will generally be denoted by a subscript $\tau$. For example, $D, D_{\lambda}$ naturally restrict

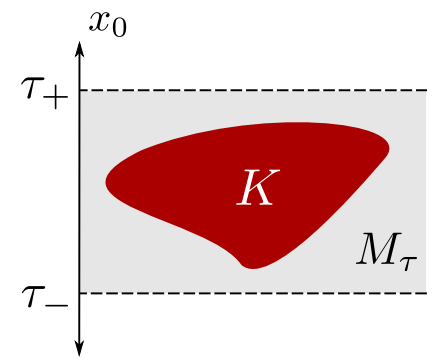
to $\mathscr{C}^{\infty}\left(M_{\tau}\right)$, and these restrictions are denoted $D_{\tau}$, $D_{\tau, \lambda}$. For the fundamental solutions $R^{ \pm}: \mathscr{C}_{0}^{\infty} \rightarrow \mathscr{C}^{\infty}$, we denote the restriction in domain and range by $R_{\tau}^{ \pm}$, i.e. $R_{\tau}^{ \pm}: \mathscr{C}_{0}^{\infty}\left(M_{\tau}\right) \rightarrow \mathscr{C}^{\infty}\left(M_{\tau}\right)$. As $W f=0$ for supp $f \cap M_{\tau}=\emptyset$, we omit the index $\tau$ when considering $W$ as restricted to $\mathscr{L}^{2}\left(M_{\tau}\right)$. Finally, the causal future/past of a set $B \subset M_{\tau}$ in $M_{\tau}$ is denoted $J_{\tau}^{ \pm}(B)=J^{ \pm}(B) \cap M_{\tau}$.

As the time slice $M_{\tau}$ is causally convex, $D 7$ ) applies, the restricted differential operator $D_{\tau}$ has the unique advanced and retarded fundamental solutions $R_{\tau}^{ \pm}$, i.e. $R_{\tau}^{ \pm}: \mathscr{C}_{0}^{\infty}\left(M_{\tau}\right) \rightarrow \mathscr{C}^{\infty}\left(M_{\tau}\right)$ are continuous and linear and satisfy $D_{\tau} R_{\tau}^{ \pm} f=f=$ $R_{\tau}^{ \pm} D_{\tau} f$ and $\operatorname{supp}\left(R_{\tau}^{ \pm} f\right) \subset J_{\tau}^{ \pm}(\operatorname{supp} f)$ for any $f \in \mathscr{C}_{0}^{\infty}\left(M_{\tau}\right)$. Due to the finite extension of $M_{\tau}$ in time direction, we have the following additional statement.

Lemma 2.5. The fundamental solutions $R_{\tau}^{ \pm}$of $D_{\tau}$ satisfy $R_{\tau}^{ \pm}\left(\mathscr{C}_{0}^{\infty}\left(M_{\tau}\right)\right) \subset$ $\mathscr{C}^{\infty}\left(M_{\tau}\right) \cap \mathscr{L}^{2}\left(M_{\tau}\right)$, and $R_{\tau}^{ \pm}: \mathscr{C}_{0}^{\infty}\left(M_{\tau}\right) \rightarrow \mathscr{L}^{2}\left(M_{\tau}\right)$ is continuous. 
Proof. Consider a compact set $B \subset M_{\tau}$. As $R_{\tau}^{ \pm}: \mathscr{C}_{0}^{\infty}\left(M_{\tau}\right) \rightarrow \mathscr{C}^{\infty}\left(M_{\tau}\right)$ is continuous, we have continuity of $R_{\tau}^{ \pm}: \mathscr{C}_{0}^{\infty}(B) \rightarrow \mathscr{C}^{\infty}\left(J_{\tau}^{ \pm}(B)\right)$. In view of the finite extension of $M_{\tau}$ in time direction, $J_{\tau}^{ \pm}(B)$ is bounded, and furthermore, for any $f \in \mathscr{C}_{0}^{\infty}(B)$, the smooth function $R_{\tau}^{ \pm} f$ extends continuously to $\overline{M_{\tau}}$. This implies that we also have a continuous inclusion $R^{ \pm} \mathscr{C}_{0}^{\infty}(B) \hookrightarrow \mathscr{L}^{2}\left(M_{\tau}\right)$. Thus $R_{\tau}^{ \pm}\left(\mathscr{C}_{0}^{\infty}\left(M_{\tau}\right)\right) \subset \mathscr{C}^{\infty}\left(M_{\tau}\right) \cap \mathscr{L}^{2}\left(M_{\tau}\right)$, the map $R_{\tau}^{ \pm}: \mathscr{C}_{0}^{\infty}(B) \rightarrow \mathscr{L}^{2}\left(M_{\tau}\right)$ is continuous, and by definition of the inductive limit topology of $\mathscr{C}_{0}^{\infty}\left(M_{\tau}\right)$, also the continuity $R_{\tau}^{ \pm}: \mathscr{C}_{0}^{\infty}\left(M_{\tau}\right) \rightarrow \mathscr{L}^{2}\left(M_{\tau}\right)$ follows.

As a consequence of this lemma, we have the following result, which will be important in the sequel.

Proposition 2.6. The operators $W R_{\tau}^{ \pm}$and $R_{\tau}^{ \pm} W$ extend from $\mathscr{C}_{0}^{\infty}\left(M_{\tau}\right)$ to bounded operators on $\mathscr{L}^{2}\left(M_{\tau}\right)$. Furthermore, $R_{\tau}^{ \pm} W\left(\mathscr{L}^{2}\left(M_{\tau}\right)\right) \subset \mathscr{C}^{\infty}\left(M_{\tau}\right) \cap \mathscr{L}^{2}\left(M_{\tau}\right)$ and $W R_{\tau}^{ \pm}\left(\mathscr{L}^{2}\left(M_{\tau}\right)\right) \subset \mathscr{C}_{0}^{\infty}(K) \subset \mathscr{C}_{0}^{\infty}\left(M_{\tau}\right)$.

Proof. By Lemma 2.5, $R_{\tau}^{ \pm}: \mathscr{C}_{0}^{\infty}\left(M_{\tau}\right) \rightarrow \mathscr{L}^{2}\left(M_{\tau}\right)$ is continuous, with image contained in $\mathscr{C}^{\infty}\left(M_{\tau}\right) \cap \mathscr{L}^{2}\left(M_{\tau}\right)$, and by the smoothing property $\left.W 2\right)$ of the perturbation, also $W: \mathscr{L}^{2}\left(M_{\tau}\right) \rightarrow \mathscr{C}_{0}^{\infty}\left(M_{\tau}\right)$ is continuous. Hence the compositions

$$
R_{\tau}^{ \pm} W: \mathscr{L}^{2}\left(M_{\tau}\right) \rightarrow \mathscr{L}^{2}\left(M_{\tau}\right), \quad W R_{\tau}^{ \pm}: \mathscr{C}_{0}^{\infty}\left(M_{\tau}\right) \rightarrow \mathscr{C}_{0}^{\infty}\left(M_{\tau}\right),
$$

are well-defined and continuous, and the first one has image contained in $\mathscr{C}^{\infty}\left(M_{\tau}\right) \cap$ $\mathscr{L}^{2}\left(M_{\tau}\right)$. Since continuity and boundedness are the same for linear maps on $\mathscr{L}^{2}\left(M_{\tau}\right)$, this already gives $\left\|R_{\tau}^{ \pm} W\right\|<\infty$, where $\|\cdot\|$ denotes the norm of $\mathcal{B}\left(\mathscr{L}^{2}\left(M_{\tau}\right)\right)$.

To show the same for $W R_{\tau}^{ \pm}$, recall that the adjoint differential operator $D_{\tau}^{*}$ also has continuous advanced and retarded fundamental solutions, which we denote here by $S_{\tau}^{ \pm}$, and which are related to $R_{\tau}^{ \pm}$by $\left(S_{\tau}^{ \pm}\right)^{*}=R_{\tau}^{\mp}$, cf. D5) and D7). Taking also into account that $W^{*} \in \mathcal{W}$, it follows as above that also $S_{\tau}^{\mp} W^{*}$ extends to a bounded operator on $\mathscr{L}^{2}\left(M_{\tau}\right)$. Thus its adjoint $\left(S_{\tau}^{\mp} W^{*}\right)^{*}=W R_{\tau}^{ \pm}$is bounded as well.

It remains to show $W R_{\tau}^{ \pm}\left(\mathscr{L}^{2}\left(M_{\tau}\right)\right) \subset \mathscr{C}_{0}^{\infty}(K)$. To this end, let $\Delta=\partial_{0}^{2}+$ $\partial_{1}^{2}+\cdots+\partial_{n-1}^{2}$ denote the Laplace operator and recall that $(1-\Delta)^{k} W$ also extends to a bounded operator $\mathscr{L}^{2}\left(M_{\tau}\right) \rightarrow \mathscr{C}_{0}^{\infty}\left(M_{\tau}\right)$, for any $k \in \mathbb{N}$ (Lemma 2.4 W4)). Thus $W R_{\tau}^{ \pm}=(1-\Delta)^{-k} \cdot(1-\Delta)^{k} W R_{\tau}^{ \pm}$maps to smooth functions. Finally, since $W f=0$ for supp $f \cap K=\emptyset$, it is also clear that the image of $W R_{\tau}^{ \pm}$consists of functions with support in $K \subset M_{\tau}$, i.e. $W R_{\tau}^{ \pm}\left(\mathscr{L}^{2}\left(M_{\tau}\right)\right) \subset \mathscr{C}_{0}^{\infty}\left(M_{\tau}\right)$.

We shall now construct advanced and retarded fundamental solutions for $D_{\tau, \lambda}$ on the time slice $M_{\tau}$. As explained earlier, such Green's operators can be expected to be of the form $\left(1+\lambda R^{ \pm} W\right)^{-1} R^{ \pm}=\sum_{k=0}^{\infty}\left(-\lambda R^{ \pm} W\right)^{k} R^{ \pm}$. Thanks to the restriction to $M_{\tau}$, the convergence of the geometric series can be controlled. It turns out to be advantageous to also discuss the series with $R^{ \pm}$and $W$ interchanged, i.e., for $\lambda \in \mathbb{C}$, 
we introduce the series expressions

$$
N_{\tau, \lambda}^{ \pm}:=\sum_{k=0}^{\infty}\left(-\lambda R_{\tau}^{ \pm} W\right)^{k}, \quad \tilde{N}_{\tau, \lambda}^{ \pm}=\sum_{k=0}^{\infty}\left(-\lambda W R_{\tau}^{ \pm}\right)^{k} .
$$

Before investigating these series further, it is worth making some remarks. First, standard theorems of functional analysis show that they converge for sufficiently small $|\lambda|$ if $\lambda W R_{\tau}^{ \pm}$and $\lambda R_{\tau}^{ \pm} W$ are bounded operators, in this case, on $\mathscr{L}^{2}\left(M_{\tau}\right)$; then the converging series also are bounded operators on $\mathscr{L}^{2}\left(M_{\tau}\right)$. In the case when $D$ is hyperbolic, one could make use of results in [1,23], establishing that $R_{\tau}^{ \pm}$ are bounded operators in $\mathscr{L}^{2}\left(M_{\tau}\right)$ on restriction to compact subsets of $M_{\tau}$, which implies $\mathscr{L}^{2}\left(M_{\tau}\right)$-boundedness of $W R_{\tau}^{ \pm}$and $R_{\tau}^{ \pm} W$ in view of $W$ having compact domain- and range-support and since $W$ is bounded as well. We shall proceed a bit differently here, also to cover the case of pre-hyperbolic $D$ as well, and will make use of the fact that $W$ is not only bounded, but also a smoothing operator, rendering $W R_{\tau}^{ \pm}$and $R_{\tau}^{ \pm} W$ bounded on $\mathscr{L}^{2}\left(M_{\tau}\right)$. The arguments we use are adapted to also showing that $\left(1+\lambda R^{ \pm} W\right)^{-1} R^{ \pm}$maps $\mathscr{C}_{0}^{\infty}\left(M_{\tau}\right)$ to $\mathscr{C}^{\infty}\left(M_{\tau}\right)$. Actually $W R_{\tau}^{ \pm}$and $R_{\tau}^{ \pm} W$ are bounded with respect to any Sobolev norm on $\mathscr{C}_{0}^{\infty}\left(M_{\tau}\right)$ owing to $W$ being a smoothing operator with compact support.

In fact, that property turns $W R_{\tau}^{ \pm}$and $R_{\tau}^{ \pm} W$ into compact operators in $\mathscr{L}^{2}\left(M_{\tau}\right)$ (and even in any Sobolev space over $M_{\tau}$ ). As a consequence, the analytic Fredholm theorem [39, Thm. VI.14] asserts that $N_{\tau, \lambda}^{ \pm}$(and similarly $\tilde{N}_{\tau, \lambda}^{ \pm}$) is a meromorphic function in $\lambda$, and is analytic in a neighborhood of $\lambda=0$ (we thank Alexander Strohmaier for pointing this out to us). We shall, however, not make use of this within the scope of the present work, where we will later mostly be interested in the first derivative of $N_{\tau, \lambda}^{ \pm} R_{\tau}^{ \pm}$with respect to $\lambda$ at $\lambda=0$.

For the series $N_{\tau, \lambda}^{ \pm}$, we next establish the following properties.

Proposition 2.7. There exists $\lambda_{0}>0$ such that for all $\lambda \in \mathbb{C}$ with $|\lambda|<\lambda_{0}$,

a) the right hand sides of (2.7) converge in the operator norm of the bounded linear operators on $\mathscr{L}^{2}\left(M_{\tau}\right)$, and therefore define bounded linear operators $N_{\tau, \lambda}^{ \pm}, \tilde{N}_{\tau, \lambda}^{ \pm}: \mathscr{L}^{2}\left(M_{\tau}\right) \rightarrow \mathscr{L}^{2}\left(M_{\tau}\right)$,

b) $N_{\tau, \lambda}^{ \pm}$and $\tilde{N}_{\tau, \lambda}^{ \pm}$are the inverse operators to $1+\lambda R_{\tau}^{ \pm} W$ and $1+\lambda W R_{\tau}^{ \pm}$, respectively (in the algebra of bounded linear operators on $\mathscr{L}^{2}\left(M_{\tau}\right)$ ), i.e.,

$$
N_{\tau, \lambda}^{ \pm}=\left(1+\lambda R_{\tau}^{ \pm} W\right)^{-1}, \quad \tilde{N}_{\tau, \lambda}^{ \pm}=\left(1+\lambda W R_{\tau}^{ \pm}\right)^{-1},
$$

c) $\tilde{N}_{\tau, \lambda}^{ \pm}$restricts to a continuous map $\tilde{N}_{\tau, \lambda}^{ \pm}: \mathscr{C}_{0}^{\infty}\left(M_{\tau}\right) \rightarrow \mathscr{C}_{0}^{\infty}\left(M_{\tau}\right)$, and $N_{\tau, \lambda}^{ \pm}\left(\mathscr{C}^{\infty}\left(M_{\tau}\right) \cap \mathscr{L}^{2}\left(M_{\tau}\right)\right) \subset \mathscr{C}^{\infty}\left(M_{\tau}\right) \cap \mathscr{L}^{2}\left(M_{\tau}\right)$.

d) For $f \in \mathscr{C}_{0}^{\infty}\left(M_{\tau}\right)$, we have $\operatorname{supp}\left(\tilde{N}_{\tau, \lambda}^{ \pm} f\right) \subset \operatorname{supp} f \cup K$. If supp $f \cap K=\emptyset$, then $N_{\tau, \lambda}^{ \pm} f=f$. 
e) For $f \in \mathscr{C}_{0}^{\infty}\left(M_{\tau}\right)$,

$$
N_{\tau, \lambda}^{ \pm} R_{\tau}^{ \pm} f=R_{\tau}^{ \pm} \tilde{N}_{\tau, \lambda}^{ \pm} f
$$

Proof. a) We have shown in Prop. 2.6 that $\lambda R_{\tau}^{ \pm} W$ and $\lambda W R_{\tau}^{ \pm}$extend to bounded linear operators on $\mathscr{L}^{2}\left(M_{\tau}\right)$. We follow the usual practice and identify these operators with their bounded extensions. For $|\lambda|<\min \left\{\left\|R_{\tau}^{ \pm} W\right\|^{-1},\left\|W R_{\tau}^{ \pm}\right\|^{-1}\right\}=: \lambda_{0}$, the operator norms of $\lambda R_{\tau}^{ \pm} W, \lambda W R_{\tau}^{ \pm}$are strictly smaller than 1 , and hence the series on the right hand sides of (2.7) converge in the operator norm. From now on, we only consider such $|\lambda|<\lambda_{0}$.

$b$ ) The series on the right hand sides of (2.7) are Neumann series and thus coincide with $\left(1+\lambda R_{\tau}^{ \pm} W\right)^{-1}$ and $\left(1+\lambda W R_{\tau}^{ \pm}\right)^{-1}$, respectively (see e.g. [9], Sec. 2.2.1).

c) We have

$$
\begin{aligned}
& N_{\tau, \lambda}^{ \pm}=1-\lambda R_{\tau}^{ \pm} W \sum_{k=0}^{\infty}\left(-\lambda R_{\tau}^{ \pm} W\right)^{k}=1-\lambda R_{\tau}^{ \pm} W N_{\tau, \lambda}^{ \pm}=1-\lambda N_{\tau, \lambda}^{ \pm} R_{\tau}^{ \pm} W \\
& \tilde{N}_{\tau, \lambda}^{ \pm}=1-\lambda W R_{\tau}^{ \pm} \sum_{k=0}^{\infty}\left(-\lambda W R_{\tau}^{ \pm}\right)^{k}=1-\lambda W R_{\tau}^{ \pm} \tilde{N}_{\tau, \lambda}^{ \pm}
\end{aligned}
$$

as operators on $\mathscr{L}^{2}\left(M_{\tau}\right)$. As $R_{\tau}^{ \pm} W\left(\mathscr{L}^{2}\left(M_{\tau}\right)\right) \subset \mathscr{C}^{\infty}\left(M_{\tau}\right) \cap \mathscr{L}^{2}\left(M_{\tau}\right)$ and $W R_{\tau}^{ \pm}\left(\mathscr{L}^{2}\left(M_{\tau}\right)\right) \subset \mathscr{C}_{0}^{\infty}\left(M_{\tau}\right)$ (Prop. 2.6), the claimed restrictions of $N_{\tau, \lambda}^{ \pm}$and $\tilde{N}_{\tau, \lambda}^{ \pm}$ follow. Furthermore, since $\mathscr{C}_{0}^{\infty}\left(M_{\tau}\right)$ is continuously embedded in $\mathscr{L}^{2}\left(M_{\tau}\right), \tilde{N}_{\tau, \lambda}^{ \pm}$is a bounded operator on $\mathscr{L}^{2}\left(M_{\tau}\right)$, and $W R_{\tau}^{ \pm}: \mathscr{L}^{2}\left(M_{\tau}\right) \rightarrow \mathscr{C}_{0}^{\infty}\left(M_{\tau}\right)$ is continuous, also the continuity of $\tilde{N}_{\tau, \lambda}^{ \pm}: \mathscr{C}_{0}^{\infty}\left(M_{\tau}\right) \rightarrow \mathscr{C}_{0}^{\infty}\left(M_{\tau}\right)$ follows.

d) This follows immediately from $(2.10,2.11)$ and the support properties $W 1)$.

$e$ ) We first note that by Lemma 2.5 and part $c$ ), the expressions $N_{\tau, \lambda}^{ \pm} R_{\tau}^{ \pm} f$ and $R_{\tau}^{ \pm} \tilde{N}_{\tau, \lambda}^{ \pm} f$ are well-defined for $f \in \mathscr{C}_{0}^{\infty}\left(M_{\tau}\right)$. Then, with arbitrary $g \in \mathscr{C}_{0}^{\infty}\left(M_{\tau}\right)$,

$$
\begin{aligned}
\left\langle g, N_{\tau, \lambda}^{ \pm} R_{\tau}^{ \pm} f\right\rangle & =\sum_{k=0}^{\infty}\left\langle g,\left(-\lambda R_{\tau}^{ \pm} W\right)^{k} R_{\tau}^{ \pm} f\right\rangle=\sum_{k=0}^{\infty}\left\langle g, R_{\tau}^{ \pm}\left(-\lambda W R_{\tau}^{ \pm}\right)^{k} f\right\rangle \\
& =\sum_{k=0}^{\infty}\left\langle\left(R_{\tau}^{ \pm}\right)^{*} g,\left(-\lambda W R_{\tau}^{ \pm}\right)^{k} f\right\rangle \\
& =\left\langle\left(R_{\tau}^{ \pm}\right)^{*} g, \tilde{N}_{\tau, \lambda}^{ \pm} f\right\rangle=\left\langle g, R_{\tau}^{ \pm} \tilde{N}_{\tau, \lambda}^{ \pm} f\right\rangle
\end{aligned}
$$

This implies (2.9). 
Proposition 2.7 puts us in the position to obtain fundamental solutions of $D_{\tau, \lambda}$. Here and in the following, we only consider $\lambda$ with

$$
|\lambda|<\min \left\{\left\|R_{\tau}^{+} W\right\|^{-1},\left\|R_{\tau}^{-} W\right\|^{-1},\left\|W R_{\tau}^{+}\right\|^{-1},\left\|W R_{\tau}^{-}\right\|^{-1}\right\},
$$

so that we can use the preceding results, and indicate that by writing "for sufficiently small $|\lambda| "$.

As an aside, we mention that this restriction on the coupling $\lambda$ can also be understood as a way of preserving the hyperbolic character of $D$. In fact, a perturbation $W \in \mathcal{W}$ with general coupling $\lambda$ can change the hyperbolic character of $D$ drastically, for example to the effect that there exist solutions of the homogeneous equation $D_{\lambda} f=0$ that have compact support.

Example 2.8 (Compactly supported solutions). Let $W$ be a $\mathscr{C}_{0}^{\infty}$-kernel operator of the form $W f=\left\langle w_{1}, f\right\rangle \cdot D w_{2}$, with $w_{1}, w_{2} \in \mathscr{C}_{0}^{\infty}$ such that $\left\langle w_{1}, w_{2}\right\rangle \neq 0$. Then there exists $\lambda \in \mathbb{C}$ such that $D_{\lambda}$ has non-zero compactly supported solutions.

Proof. One computes $(D+\lambda W) f=D f+\lambda\left\langle w_{1}, f\right\rangle \cdot D w_{2}$, and this expression vanishes for $f=w_{2} \in \mathscr{C}_{0}^{\infty}$ and $\lambda=-\left\langle w_{1}, w_{2}\right\rangle^{-1}$.

If compactly supported solutions exist, there can be no unique fundamental solutions, and also quantization will be ambiguous. However, these compactly supported solutions do not exist for sufficiently small $|\lambda|$.

Lemma 2.9. Let $|\lambda|$ be sufficiently small, and $f \in \mathscr{C}_{0}^{\infty}\left(M_{\tau}\right)$. If $D_{\tau, \lambda} f=0$, then $f=0$.

Proof. By assumption, we have $D_{\tau} f=-\lambda W f$, with $f \in \mathscr{C}_{0}^{\infty}\left(M_{\tau}\right)$. Applying $R_{\tau}^{ \pm}$ therefore gives $R_{\tau}^{ \pm} D_{\tau} f=f=-\lambda R_{\tau}^{ \pm} W f$, i.e. either the $\mathscr{L}^{2}\left(M_{\tau}\right)$-operator $-\lambda R_{\tau}^{ \pm} W$ has the eigenvalue 1 , or $f=0$. But we fixed $\lambda$ in such a way that $\left\|-\lambda R_{\tau}^{ \pm} W\right\|<1$. Hence $f=0$.

After this remark, we proceed to the fundamental solutions of $D_{\tau, \lambda}$, and introduce the operators

$$
R_{\tau, \lambda}^{ \pm}:=N_{\tau, \lambda}^{ \pm} R_{\tau}^{ \pm}=R_{\tau}^{ \pm} \tilde{N}_{\tau, \lambda}^{ \pm}: \mathscr{C}_{0}^{\infty}\left(M_{\tau}\right) \rightarrow \mathscr{C}^{\infty}\left(M_{\tau}\right) \cap \mathscr{L}^{2}\left(M_{\tau}\right),
$$

which are well defined by the properties of $R_{\tau}^{ \pm}$(Lemma 2.5) and $N_{\tau, \lambda}^{ \pm}, \tilde{N}_{\tau, \lambda}^{ \pm}$ (Prop. 2.7). By Prop. $2.7 c$ ), they are also continuous as maps $\mathscr{C}_{0}^{\infty}\left(M_{\tau}\right) \rightarrow \mathscr{C}^{\infty}\left(M_{\tau}\right)$.

Theorem 2.10 (Fundamental solutions on a time slice). For sufficiently small $|\lambda|$, the operators $R_{\tau, \lambda}^{ \pm}: \mathscr{C}_{0}^{\infty}\left(M_{\tau}\right) \rightarrow \mathscr{C}^{\infty}\left(M_{\tau}\right)$ (2.12) exist as continuous linear maps and satisfy, $f, g \in \mathscr{C}_{0}^{\infty}\left(M_{\tau}\right)$

a) $D_{\tau, \lambda} R_{\tau, \lambda}^{ \pm} f=f=R_{\tau, \lambda}^{ \pm} D_{\tau, \lambda} f$.

b) $\operatorname{supp}\left(R_{\tau, \lambda}^{ \pm} f\right) \subset J_{\tau}^{ \pm}(\operatorname{supp} f) \cup J_{\tau}^{ \pm}(K)$. 
c) $\operatorname{supp}\left(R_{\tau, \lambda}^{ \pm} f-R_{\tau}^{ \pm} f\right) \subset J_{\tau}^{ \pm}(K)$.

d) If $J_{\tau}^{ \pm}(\operatorname{supp} f) \cap K=\emptyset$, then

$$
R_{\tau, \lambda}^{ \pm} f=R_{\tau}^{ \pm} f
$$

e) If $D$ and $W$ are symmetric, i.e. $D=D^{*}, W=W^{*}$, and $\lambda \in \mathbb{R}$, then one has, $f, g \in \mathscr{C}_{0}^{\infty}\left(M_{\tau}\right)$

$$
\left\langle g, R_{\tau, \lambda}^{ \pm} f\right\rangle=\left\langle R_{\tau, \lambda}^{\mp} g, f\right\rangle .
$$

f) If $\tau=\left(\tau_{-}, \tau_{+}\right)$is replaced by $\tau^{\prime}=\left(\tau_{-}^{\prime}, \tau_{+}^{\prime}\right)$, with $\tau_{-}^{\prime}>\tau_{-}$and $\tau_{+}^{\prime}<\tau_{+}$ such that $K \subset M_{\tau^{\prime}} \subset M_{\tau}$, the statements a)-e) still hold.

Proof. a) Note that the perturbed differential operator $D_{\tau, \lambda}$, which is defined on $\mathscr{C}^{\infty}\left(M_{\tau}\right)$, restricts to $\mathscr{C}_{0}^{\infty}\left(M_{\tau}\right)$ by the properties of $D_{\tau}$ and $W$. Hence both compositions, $D_{\tau, \lambda} R_{\tau, \lambda}^{ \pm}$and $R_{\tau, \lambda}^{ \pm} D_{\tau, \lambda}$, are well-defined on $\mathscr{C}_{0}^{\infty}\left(M_{\tau}\right)$.

For $f \in \mathscr{C}_{0}^{\infty}\left(M_{\tau}\right)$, we compute

$$
\begin{aligned}
R_{\tau, \lambda}^{ \pm} D_{\tau, \lambda} f & =N_{\tau, \lambda}^{ \pm} R_{\tau}^{ \pm}\left(D_{\tau}+\lambda W\right) f \\
& =\left(1+\lambda R_{\tau}^{ \pm} W\right)^{-1}\left(f+\lambda R_{\tau}^{ \pm} W f\right)=f,
\end{aligned}
$$

where we have used that $R_{\tau}^{ \pm}$is a fundamental solution of $D_{\tau}$, i.e. $R_{\tau}^{ \pm} D_{\tau} f=f$. Similarly,

$$
\begin{aligned}
D_{\tau, \lambda} R_{\tau, \lambda}^{ \pm} f & =\left(D_{\tau}+\lambda W\right) R_{\tau}^{ \pm} \tilde{N}_{\tau, \lambda}^{ \pm} f=\tilde{N}_{\tau, \lambda}^{ \pm} f+\lambda W R_{\tau}^{ \pm} \tilde{N}_{\tau, \lambda}^{ \pm} f \\
& =\left(1+\lambda W R_{\tau}^{ \pm}\right)\left(1+\lambda W R_{\tau}^{ \pm}\right)^{-1} f=f .
\end{aligned}
$$

$b$ ), c), d): Using the hyperbolic character of $R_{\tau}^{ \pm}$and Prop. $2.7 d$ ), we get $\operatorname{supp}\left(R_{\tau, \lambda}^{ \pm} f\right)=\operatorname{supp}\left(R_{\tau}^{ \pm} \tilde{N}_{\tau, \lambda}^{ \pm} f\right) \subset J_{\tau}^{ \pm}(\operatorname{supp} f \cup K)=J_{\tau}^{ \pm}(\operatorname{supp} f) \cup J_{\tau}^{ \pm}(K)$. Replacing $f$ by $R_{\tau, \lambda}^{ \pm} f$ in the second statement of Prop. $2.7 d$ ), we also get $R_{\tau, \lambda}^{ \pm} f=$ $N_{\tau, \lambda}^{ \pm} R_{\tau}^{ \pm} f=R_{\tau}^{ \pm} f$ in case $\operatorname{supp}\left(R_{\tau}^{ \pm} f\right) \subset J_{\tau}^{ \pm}(\operatorname{supp} f)$ is disjoint from $K$, i.e. eqn. (2.13). For $c$ ), observe that by (2.11)

$$
R_{\tau}^{ \pm} f-R_{\tau, \lambda}^{ \pm} f=R_{\tau}^{ \pm}\left(1-\tilde{N}_{\tau, \lambda}^{ \pm}\right) f=\lambda R_{\tau}^{ \pm} W R_{\tau}^{ \pm} \tilde{N}_{\tau, \lambda}^{ \pm} f
$$

As $W R_{\tau}^{ \pm} \tilde{N}_{\tau, \lambda}^{ \pm} f$ has support in $K$, we get $\operatorname{supp}\left(R_{\tau}^{ \pm} f-R_{\tau, \lambda}^{ \pm} f\right) \subset J_{\tau}^{ \pm}(K)$ as claimed.

e) Using the symmetry of $D$, and thus of $D_{\tau}$, we have $\left(R_{\tau}^{ \pm}\right)^{*}=R_{\tau}^{\mp}$. With $W=W^{*}$, this gives

$$
\begin{aligned}
\left\langle g, R_{\tau, \lambda}^{ \pm}, f\right\rangle & =\sum_{k=0}^{\infty}\left\langle g, R_{\tau}^{ \pm}\left(-\lambda W R_{\tau}^{ \pm}\right)^{k} f\right\rangle \\
& =\sum_{k=0}^{\infty}\left\langle\left(-\lambda R_{\tau}^{\mp} W\right)^{k} R_{\tau}^{\mp} g, f\right\rangle=\left\langle R_{\tau, \lambda}^{\mp} g, f\right\rangle .
\end{aligned}
$$


f) The operator norms of the restrictions of the bounded operators $R_{\tau}^{ \pm} W, W R_{\tau}^{ \pm}$ from $\mathscr{L}^{2}\left(M_{\tau}\right)$ to the subspace $\mathscr{L}^{2}\left(M_{\tau^{\prime}}\right)$ are not larger than the norms of their unrestricted counterparts. Thus any $|\lambda|$ that is sufficiently small for the time cutoff $\tau$ is also sufficiently small for the sharper time cutoff $\tau^{\prime}$, and hence $a$ ) $-e$ ) remain valid for $\tau^{\prime}$ instead of $\tau$.

This theorem shows that the $R_{\tau, \lambda}^{ \pm}$are quite close to advanced/retarded fundamental solutions, with possible acausal propagation in the future/past of the perturbation region $K$. Despite these differences to advanced/retarded fundamental solutions for local differential operators, we will refer to the $R_{\tau, \lambda}^{ \pm}$with the same terminology as in the local case.

Defining $R_{\tau, \lambda}:=R_{\tau, \lambda}^{-}-R_{\tau, \lambda}^{+}$, it is clear from part $a$ ) of the theorem that any function of the form $R_{\tau, \lambda} g, g \in \mathscr{C}_{0}^{\infty}\left(M_{\tau}\right)$, is a solution of $D_{\tau, \lambda}$.

In the next step we will extend the fundamental solutions in the time slice $M_{\tau}$ to all of $\mathbb{R}^{n}$. As the potential $W$ vanishes outside $M_{\tau}$, this amounts to a "gluing" of advanced/retarded solutions of $D f=0$ outside $M_{\tau}$ with advanced/retarded solutions of $D_{\tau, \lambda} f=0$ in $M_{\tau}$.

We first introduce some notation. Let $\varepsilon>0$, and define

$$
\begin{aligned}
& M_{\tau, \varepsilon}^{-}:=\Sigma_{\tau_{-}}^{+} \cap \Sigma_{\tau_{-}+\varepsilon}^{-}, \\
& M_{\tau, \varepsilon}^{+}:=\Sigma_{\tau_{+}-\varepsilon}^{+} \cap \Sigma_{\tau_{+}}^{-}, \\
& M_{\tau, \varepsilon}:=\Sigma_{\tau_{-}+\varepsilon}^{+} \cap \Sigma_{\tau_{+}-\varepsilon}^{-},
\end{aligned}
$$

We require that $\varepsilon$ is so small that $K \subset M_{\tau, \varepsilon}$, as depicted in the figure on the right.

Given $h \in \mathscr{C}_{0}^{\infty}\left(M_{\tau, \varepsilon}\right)$, the function $R_{\tau, \lambda}^{+} h$

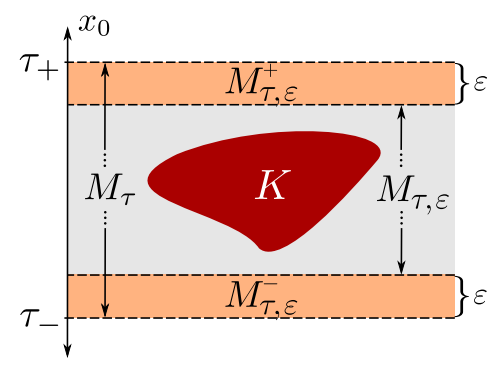
vanishes on $M_{\tau, \varepsilon}^{-}$, and is a solution of $D$ with compactly supported Cauchy data on $M_{\tau, \varepsilon}^{+}-$ this follows from Thm. $2.10 \mathrm{~b}$ ) and the fact that $K$ does not intersect $M_{\tau, \varepsilon}^{ \pm}$. We denote its Cauchy data on $\Sigma_{\tau_{+}-\varepsilon}$ by $u_{h}^{+}$. Similarly, $R_{\tau, \lambda}^{-} h$ vanishes on $M_{\tau, \varepsilon}^{+}$, and is a solution of $D$ with compactly supported Cauchy data on $M_{\tau, \varepsilon}^{-}$; its Cauchy data on $\Sigma_{\tau_{-}+\varepsilon}$ will be denoted $u_{h}^{-}$.

Given Cauchy data $u$ on some Cauchy hyperplane $\Sigma$, the corresponding solution of $D$ will always be denoted $f_{0}[u]$. We define

$$
\left(R_{\lambda}^{ \pm} h\right)(x):= \begin{cases}\left(R_{\tau, \lambda}^{ \pm} h\right)(x) & x \in M_{\tau} \\ f_{0}\left[u_{h}^{ \pm}\right](x) & x \in \Sigma_{\tau_{ \pm} \mp \varepsilon}^{ \pm}, \quad h \in \mathscr{C}_{0}^{\infty}\left(M_{\tau, \varepsilon}\right) . \\ 0 & x \in \Sigma_{\tau_{\mp} \pm \varepsilon}^{\mp}\end{cases}
$$

This assignment is well-defined in the overlap regions $M_{\tau, \varepsilon}^{+}$and $M_{\tau, \varepsilon}^{-}$. In fact, $\left(R_{\tau, \lambda}^{ \pm} h\right)(x)=0$ for $x \in M_{\tau, \varepsilon}^{\mp}$ as recalled above, and $R_{\tau, \lambda} h$ is a solution of $D$ 
on the strip $M_{\tau, \varepsilon}^{ \pm}$. As this solution is uniquely fixed by its Cauchy data, it coincides with $f_{0}\left[u_{h}^{ \pm}\right]$in this region.

It is also clear that (2.15) restricts to $R_{\tau, \lambda}^{ \pm} h$ on $M_{\tau}$, and is an advanced/retarded fundamental solution of $D_{\lambda}$ in the sense that $D_{\lambda} R_{\lambda}^{ \pm} h=h=R_{\lambda}^{ \pm} D_{\lambda} h$, and $\operatorname{supp}\left(R_{\lambda}^{ \pm} h\right) \subset J^{ \pm}(\operatorname{supp} h) \cup J^{ \pm}(K)$ - the latter statement is a consequence of Thm. $2.10 b$ ) and $D 4$ ). Also the items $c$ ) $-d$ ) of Thm. 2.10 hold for $R_{\lambda}^{ \pm} h$ when the index $\tau$ is dropped and $M_{\tau}$ is replaced by $\mathbb{R}^{n}$ throughout.

In a similar fashion, we now want to define $R_{\lambda}^{ \pm}$on functions $h \in \mathscr{C}_{0}^{\infty}$ whose support lies outside of $M_{\tau}$. For supp $h \subset \Sigma_{\tau_{ \pm}}^{ \pm}$, the function $R^{ \pm} h$ vanishes on $M_{\tau}$, and we therefore simply set

$$
R_{\lambda}^{ \pm} h:=R^{ \pm} h, \quad h \in \mathscr{C}_{0}^{\infty}\left(\Sigma_{\tau_{ \pm}}^{ \pm}\right)
$$

To define $R_{\lambda}^{\mp} h, h \in \mathscr{C}_{0}^{\infty}\left(\Sigma_{\tau_{ \pm}}^{ \pm}\right)$, we observe that $R^{\mp} h$ is a solution of $D$ in the strip $M_{\tau, \varepsilon}^{ \pm}$. This solution has compactly supported Cauchy data, and according to $D 6$ ), we may therefore represent it in the form $\left(R^{\mp} h\right)(x)=$ $\pm\left(R g^{ \pm}\right)(x), x \in M_{\tau, \varepsilon}^{ \pm}$, for some $g^{ \pm} \in \mathscr{C}_{0}^{\infty}$ which is supported in the strip of half the width,

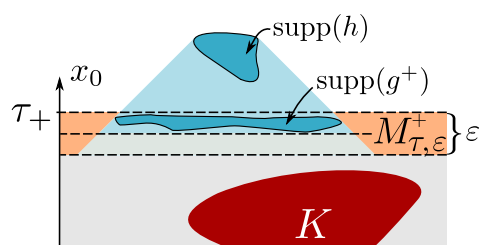
$\operatorname{supp}\left(g^{ \pm}\right) \subset M_{\tau, \varepsilon / 2}^{ \pm}$. On the inner half of $M_{\tau, \varepsilon}^{ \pm}$, we then have $\left(R^{\mp} h\right)(x)= \pm\left(R g^{ \pm}\right)(x)=\left(R^{\mp} g^{ \pm}\right)(x)=\left(R_{\tau, \lambda}^{\mp} g^{ \pm}\right)(x)$. This implies that

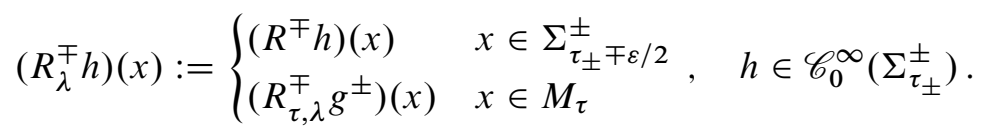

is well-defined. It remains to define $\left(R_{\lambda}^{\mp} h\right)(x)$ for $x \in \Sigma_{\tau \mp}^{\mp}$. To do so, we proceed as in the definition of $R_{\lambda}^{ \pm} h$ for $h \in \mathscr{C}_{0}^{\infty}\left(M_{\tau}\right)$, and let $v$ denote the Cauchy data of $R_{\tau, \lambda}^{\mp} g^{ \pm}$on the Cauchy hyperplane $\Sigma_{\tau \mp \pm \varepsilon}$. Then we set

$$
\left(R_{\lambda}^{\mp} h\right)(x):=f_{0}[v](x), \quad x \in \Sigma_{\tau_{\mp} \pm \varepsilon}^{\mp}, h \in \mathscr{C}_{0}^{\infty}\left(\Sigma_{\tau_{ \pm}}^{ \pm}\right) .
$$

As before, the assignment is well-defined in the overlap region, and completes our definition of $R_{\lambda}^{\mp} h$. By construction, it is clear that again the statements Thm. 2.10 $a)-d$ ) hold for $R_{\lambda}^{ \pm} h$ when the index $\tau$ is dropped and $M_{\tau}$ is replaced by $\mathbb{R}^{n}$.

Making use of Thm. $2.10 e$ ), it also becomes apparent that our construction is independent of $\varepsilon$, and also results in the same definition of $R_{\lambda}^{ \pm} h$ when $\tau$ is replaced by a sharper cut-off $\tau^{\prime}$ such that $\tau_{+}^{\prime}<\tau_{+}, \tau_{-}^{\prime}>\tau_{-}, K \subset M_{\tau^{\prime}}$. We can thus proceed to the definition of $R_{\lambda}^{ \pm} h$ for $h \in \mathscr{C}_{0}^{\infty}$ of arbitrary support with the help of a smooth partition of unity. In fact, let $1=\chi_{+}+\chi_{0}+\chi_{-}$be a smooth partition of unity, where $\chi_{ \pm}, \chi_{0}$ are smooth functions on $\mathbb{R}$ with supports supp $\chi_{+} \subset\left(\tau_{+}-\varepsilon, \infty\right)$, 
supp $\chi_{0} \subset\left(\tau_{-}, \tau_{+}\right)$, supp $\chi_{-} \subset\left(-\infty, \tau_{-}+\varepsilon\right)$. Denoting the multiplication operators with $\chi_{ \pm}\left(x_{0}\right), \chi_{0}\left(x_{0}\right)$ by the same letters, we then set

$$
R_{\lambda}^{ \pm} h:=R_{\lambda}^{ \pm} \chi_{+} h+R_{\lambda}^{ \pm} \chi_{0} h+R_{\lambda}^{ \pm} \chi_{-} h, \quad h \in \mathscr{C}_{0}^{\infty} .
$$

All functions on the right hand side have been defined before, and the left hand side inherits properties $a)-d$ ) of Thm. 2.10 from them. Finally, also Thm. $2.10 e$ ) transports to the global case: For $D=D^{*}, W=W^{*}, \lambda \in \mathbb{R}$, the integral $\left\langle f, R_{\lambda}^{ \pm} g\right\rangle$, with $f, g \in \mathscr{C}_{0}^{\infty}$, can be split in two parts, namely one integral over $M_{\tau}$ and one integral over $\mathbb{R}^{n} \backslash M_{\tau}$. On $M_{\tau}, R_{\lambda}^{ \pm}$restrict to $R_{\tau, \lambda}^{ \pm}$, and we can use Thm. $2.10 e$ ) to compute the adjoint. On the complement, the same conclusion follows from exploiting the properties of $R^{ \pm}$.

We summarize the results of our construction in the following theorem.

Theorem 2.11 (Global fundamental solutions). For sufficiently small $|\lambda|$, the operators $R_{\lambda}^{ \pm}: \mathscr{C}_{0}^{\infty} \rightarrow \mathscr{C}^{\infty}$ defined above exist as continuous linear maps and satisfy, $f, g \in \mathscr{C}_{0}^{\infty}$,

a) $D_{\lambda} R_{\lambda}^{ \pm} f=f=R_{\lambda}^{ \pm} D_{\lambda} f$.

b) $\operatorname{supp}\left(R_{\lambda}^{ \pm} f\right) \subset J^{ \pm}(\operatorname{supp} f) \cup J^{ \pm}(K)$.

c) $\operatorname{supp}\left(R_{\lambda}^{ \pm} f-R^{ \pm} f\right) \subset J^{ \pm}(K)$.

d) If $J^{ \pm}(\operatorname{supp} f) \cap K=\emptyset$, then $R_{\lambda}^{ \pm} f=R^{ \pm} f$.

e) If $D$ and $W$ are symmetric, i.e. $D=D^{*}, W=W^{*}$, and $\lambda \in \mathbb{R}$, then one has, $f, g \in \mathscr{C}_{0}^{\infty}$,

$$
\left\langle g, R_{\lambda}^{ \pm} f\right\rangle=\left\langle R_{\lambda}^{\mp} g, f\right\rangle .
$$

Remark. In [19] it was shown that there exist parametrices $F_{\lambda}, G_{\lambda}$ for $D_{\lambda}$, i.e. $F_{\lambda} D_{\lambda}=1+A_{\lambda}, D_{\lambda} G_{\lambda}=1+B_{\lambda}$, where $A_{\lambda}, B_{\lambda}$ are smoothing operators. Using this, a variation of the previous arguments shows (as is well-known) that on small enough domains, one obtains Greens-type operators $\left(1+A_{\lambda}\right)^{-1} F_{\lambda}$ and $G_{\lambda}\left(1+B_{\lambda}\right)^{-1}$ for $D_{\lambda}$. However, our arguments allow a global construction of the advanced and retarded fundamental solutions and control of their causal support properties.

According to Theorem 2.11, the influence of the perturbation $W$ is confined to the future/past of its support $K$, as if a source $u$ with support in $K$ would have been added to the unperturbed equation, i.e. $D f=u$. However, in contrast to the solutions of $D f=u$, the solutions of $D_{\lambda} f=0$ do not differ from the ones of $D f=0$ in case $J^{ \pm}(\operatorname{supp} f) \cap K=\emptyset$, i.e. if the unperturbed wave does not collide with the potential in $K$, as depicted in the figure below. 


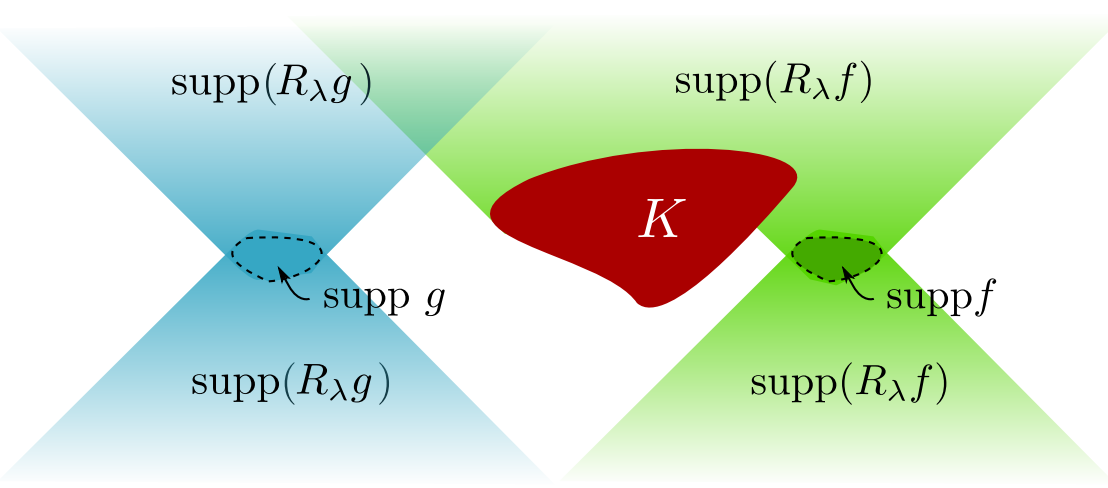

Figure 1. Typical supports of two solutions $R_{\lambda} f, R_{\lambda} g$ of $D_{\lambda}$.

So far we made no claim concerning uniqueness of the fundamental solutions, and in fact, a couple of choices were made in the construction of the $R_{\lambda}^{ \pm}$. However, we will show below that the $R_{\lambda}^{ \pm}$are in fact uniquely determined by properties $a$ ) and $b$ ) of the preceding theorem, and in particular independent of the choices made.

\section{Proposition 2.12 (Uniqueness properties).}

a) Let $f \in \mathscr{C}^{\infty}$ satisfy $D_{\lambda} f=0$ and $\operatorname{supp} f \subset V^{+}+a$ or $\operatorname{supp} f \subset V^{-}+a$ for some $a \in \mathbb{R}^{n}$. Then $f=0$.

b) The advanced/retarded fundamental solutions $R_{\lambda}^{ \pm}$are the unique linear maps $\mathscr{C}_{0}^{\infty} \rightarrow \mathscr{C}^{\infty}$ satisfying properties a) and b) of Theorem 2.11.

Proof. a) This argument is based on Green's second identity (or Gauss' theorem), and we will have to distinguish the cases where $D$ is normally hyperbolic and prenormally hyperbolic, respectively. We begin with the normally hyperbolic case, i.e. $D=\square+U^{\mu}(x) \partial_{\mu}+V(x)$, where $U^{\mu}, V: \mathbb{R}^{n} \rightarrow \mathbb{C}^{N \times N}$ are smooth functions.

Let $f$ be a solution of $D_{\lambda}, h \in \mathscr{C}_{0}^{\infty}$, and $\Sigma_{t}$ a Cauchy hyperplane. We set $g_{\lambda}^{ \pm}:=T_{\lambda}^{\mp} h$, where $T_{\lambda}^{\mp}=\left(R_{\lambda}^{ \pm}\right)^{*}$ are the advanced/retarded fundamental solutions of $D_{\lambda}^{*}=D^{*}+\bar{\lambda} W^{*}$, so that the support of $x \mapsto\left(g^{ \pm}(x), \partial_{\mu} f(x)\right)$ has compact intersection with $\Sigma^{ \pm}$. We can thus use Green's second identity and integration by parts to compute

$$
\begin{aligned}
\int_{\Sigma_{t}^{ \pm}}\left(\left(D^{*} g^{ \pm}, f\right)-\left(g^{ \pm}, D f\right)\right) \\
\quad=\int_{\Sigma_{t}^{ \pm}}\left(\left(\square g^{ \pm}, f\right)-\left(g^{ \pm}, \square f\right)\right)-\int_{\Sigma_{t}^{ \pm}}\left(\left(\partial_{\mu} \overline{U^{\mu}} g^{ \pm}, f\right)-\left(g^{ \pm}, U^{\mu} \partial_{\mu} f\right)\right) \\
\quad=\mp \int_{\Sigma_{t}}\left\{\left(\partial_{0} g_{t}^{ \pm}, f_{t}\right)-\left(g_{t}^{ \pm}, \partial_{0} f_{t}\right)\right\} \pm \int_{\Sigma_{t}}\left(g_{t}^{ \pm},\left(U^{0} f\right)_{t}\right),
\end{aligned}
$$


where an index $t$ denotes restriction to $\Sigma_{t}$. The left hand side can also be evaluated by using the equations $D f=-\lambda W f$ (since $f$ is a solution) and $D^{*} g^{ \pm}=$ $h-\bar{\lambda} W^{*} g^{ \pm}$(by definition of $g^{ \pm}$),

$$
\begin{aligned}
\int_{\Sigma_{t}^{ \pm}}\left(\left(D^{*} g^{ \pm}, f\right)\right. & \left.-\left(g^{ \pm}, D f\right)\right) \\
& =\int_{\Sigma_{t}^{ \pm}}(h, f)-\lambda \int_{\Sigma_{t}^{ \pm}}\left(W^{*} T_{\lambda}^{\mp} h, f\right)+\lambda \int_{\Sigma_{t}^{ \pm}}\left(T_{\lambda}^{\mp} h, W f\right) .
\end{aligned}
$$

Adding the equations for both choices of " \pm " then gives

$$
\begin{aligned}
\langle h, f\rangle-\lambda\left\{\int_{\Sigma_{t}^{+}}\left(W^{*} T_{\lambda}^{-} h, f\right)\right. & +\int_{\Sigma_{t}^{-}}\left(W^{*} T_{\lambda}^{+} h, f\right) \\
& \left.-\int_{\Sigma_{t}^{+}}\left(T_{\lambda}^{-} h, W f\right)-\int_{\Sigma_{t}^{-}}\left(T_{\lambda}^{+} h, W f\right)\right\} \\
= & \int_{\Sigma_{t}}\left\{\left(\left(\partial_{0} R_{\lambda}^{*} h\right)_{t}, f_{t}\right)-\left(\left(R_{\lambda}^{*} h\right)_{t},\left(\partial_{0} f\right)_{t}\right)\right\}-\int_{\Sigma_{t}}\left(\left(R_{\lambda}^{*} h\right)_{t}, U_{t}^{0} f_{t}\right) .
\end{aligned}
$$

Suppose now that supp $f \subset V^{+}+a$ or supp $f \subset V^{-}+a$ for some $a \in \mathbb{R}^{n}$. Then we can choose $\Sigma_{t}$ in such a way that $K \subset \Sigma_{t}^{ \pm}$and $f_{t}=0,\left(\partial_{0} f\right)_{t}=0$. In this situation, the right hand side of the above equation vanishes, and the four terms in curly brackets cancel because in each of these integrals, the range of integration can be taken as $\mathbb{R}^{n}$ instead of $\Sigma_{t}^{ \pm}$. Hence we arrive at $\langle h, f\rangle=0$. As $h \in \mathscr{C}_{0}^{\infty}$ was arbitrary, this implies $f=0$.

For the case that $D$ is prenormally hyperbolic, we find another prenormally hyperbolic $D^{\prime}$ such that $D^{\prime} D$ is again normally hyperbolic. Moreover, by Lemma 2.4 $W 4)$, also $D^{\prime} W \in \mathcal{W}$. Thus, if $f_{\lambda}$ is a solution of $D_{\lambda}$, then $0=D^{\prime} D_{\lambda} f_{\lambda}=\left(D^{\prime} D+\right.$ $\left.\lambda D^{\prime} W\right) f_{\lambda}$, i.e. $f_{\lambda}$ is also a solution of the normally hyperbolic operator $D^{\prime} D$, perturbed by $\lambda D^{\prime} W \in \mathcal{W}$. By our previous argument for normally hyperbolic operators, we then see that $f_{\lambda}=0$ if $f_{\lambda}$ has support in a light cone.

b) If $\tilde{R}_{\lambda}^{ \pm}$is another linear map satisfying Thm. 2.11a),b), then for any $f \in \mathscr{C}_{0}^{\infty}$, the function $R_{\lambda}^{ \pm} f-\tilde{R}_{\lambda}^{ \pm} f$ is a solution of $D_{\lambda}$ (because of Thm. 2.11a)) with support in a future/past light cone (because of Thm. $2.11 b$ )). Hence $R_{\lambda}^{ \pm} f=\tilde{R}_{\lambda}^{ \pm} f$ by part $a$ ).

Having established the basic existence and uniqueness theorem on fundamental solutions, we introduce in complete analogy to the unperturbed case the space of all solutions of $D_{\lambda}$ with compactly supported Cauchy data as

$$
\begin{array}{r}
\operatorname{Sol}_{\lambda}:=\left\{f \in \mathscr{C}^{\infty}: D_{\lambda} f=0, \operatorname{supp} f \subset\left(V^{+}+a_{+}\right) \cup\left(V^{-}+a_{-}\right)\right. \\
\text {for some } \left.a_{ \pm} \in \mathbb{R}^{n}\right\},
\end{array}
$$


and define the propagator as

$$
R_{\lambda}:=R_{\lambda}^{-}-R_{\lambda}^{+}
$$

\section{Proposition 2.13 (Structure of the solution spaces).}

a) Let $\Sigma$ denote an open causally convex neighborhood of a Cauchy hyperplane $\Sigma$ such that $K \subset J^{+}(\Sigma) \backslash \Sigma$ or $K \subset J^{-}(\Sigma) \backslash \Sigma$. Then

$$
\operatorname{Sol}_{\lambda}=R_{\lambda} \mathscr{C}_{0}^{\infty}(\Sigma) \text {. }
$$

b) We have $\operatorname{ker} R_{\lambda}=D_{\lambda} \mathscr{C}_{0}^{\infty}$, and hence $\operatorname{Sol}_{\lambda} \cong \mathscr{C}_{0}^{\infty} / \operatorname{ker} R_{\lambda}=\mathscr{C}_{0}^{\infty} / D_{\lambda} \mathscr{C}_{0}^{\infty}$.

c) If $D=D^{*}, W=W^{*}$, and $\lambda \in \mathbb{R}$, the map

$$
\begin{aligned}
\rho_{\lambda}: \operatorname{Sol}_{\lambda} \times \operatorname{Sol}_{\lambda} & \rightarrow \mathbb{C}, \\
\left(R_{\lambda} f, R_{\lambda} g\right) & \mapsto\left\langle f, R_{\lambda} g\right\rangle
\end{aligned}
$$

is a well-defined non-degenerate sesquilinear form satisfying

$$
\overline{\rho_{\lambda}\left(R_{\lambda} f, R_{\lambda} g\right)}=-\rho_{\lambda}\left(R_{\lambda} g, R_{\lambda} f\right), \quad f, g \in \mathscr{C}_{0}^{\infty} .
$$

Proof. a) We carry out the proof for the case $K \subset J^{+}(\Sigma) \backslash \Sigma$, the other case is analogous. Let $f_{\lambda} \in \operatorname{Sol}_{\lambda}$ and consider the restriction $\left.f_{\lambda}\right|_{\Sigma}$. As $\Sigma$ is disjoint from $K$, this restriction is a solution of $D$ on $\Sigma$, and thus there exists $g \in \mathscr{C}_{0}^{\infty}(\Sigma)$ such that $\left.f_{\lambda}\right|_{\Sigma}=\left.(R g)\right|_{\Sigma}=\left.\left(R_{\lambda} g\right)\right|_{\Sigma}$. Hence the two solutions $f_{\lambda}, R_{\lambda} g$ of $D_{\lambda}$ coincide on $\Sigma$, i.e. $f_{\lambda}-R_{\lambda} g=h^{+}+h^{-}$, where $h^{ \pm} \in \mathscr{C}^{\infty}$ have support in $J^{ \pm}(\Sigma) \backslash \Sigma$. As $K \subset J^{+}(\Sigma) \backslash \Sigma$, both, $h^{+}$and $h^{-}$, are solutions of $D_{\lambda}$, and in view of the support properties of $f_{\lambda}$ (see (2.22)) and $R_{\lambda} g$ (see Thm. $\left.2.11 b\right)$ ), we have supp $h^{ \pm} \subset V^{ \pm}+a_{ \pm}$for some $a_{ \pm} \in \mathbb{R}^{n}$. Thus, by Proposition $2.12 a$ ), $h^{+}=h^{-}=0$, and $f_{\lambda}=R_{\lambda} g$.

b) By Theorem $2.11 a$ ), we have $R_{\lambda} D_{\lambda} f=0$ for any $f \in \mathscr{C}_{0}^{\infty}$, i.e. $D_{\lambda} \mathscr{C}_{0}^{\infty} \subset$ $\operatorname{ker} R_{\lambda}$. Conversely, for $f \in \operatorname{ker} R_{\lambda}$, the function $g:=R_{\lambda}^{+} f=R_{\lambda}^{-} f$ has compact support in view of Theorem $2.11 \mathrm{~b}$ ). Thus $f=D_{\lambda} R_{\lambda}^{+} f=D_{\lambda} g \in D_{\lambda} \mathscr{C}_{0}^{\infty}$, i.e. we have shown ker $R_{\lambda}=D_{\lambda} \mathscr{C}_{0}^{\infty}$. Now, by part $a$ ), we know $\operatorname{Sol}_{\lambda}=R_{\lambda} \mathscr{C}_{0}^{\infty}$, and thus $\operatorname{Sol}_{\lambda} \cong \mathscr{C}_{0}^{\infty} / \operatorname{ker} R_{\lambda}=\mathscr{C}_{0}^{\infty} / D_{\lambda} \mathscr{C}_{0}^{\infty}$.

c) For $f, g \in \mathscr{C}_{0}^{\infty}$, we have by Theorem $2.11 e$ )

$$
\left\langle f, R_{\lambda} g\right\rangle=\left\langle f,\left(R_{\lambda}^{-}-R_{\lambda}^{+}\right) g\right\rangle=\left\langle\left(R_{\lambda}^{+}-R_{\lambda}^{-}\right) f, g\right\rangle=-\left\langle R_{\lambda} f, g\right\rangle,
$$

and thus the assignment (2.26) is well-defined. Sesquilinearity and non-degenerateness is clear, and (2.27) follows directly from (2.28).

Next we describe the solutions of $D_{\lambda}$ in a little more detail. This is a direct corollary of our preceding constructions. 


\section{Corollary 2.14.}

a) Let $\Sigma$ be a Cauchy hyperplane such that $K \subset \Sigma^{+}$or $K \subset \Sigma^{-}$, and $u$ (smooth, compactly supported) Cauchy data on $u$. Then there exists precisely one solution $f_{\lambda} \in \operatorname{Sol}_{\lambda}$ with Cauchy data $u$ on $\Sigma$.

b) Let $f_{\lambda} \in \operatorname{Sol}_{\lambda}$ be a solution of $D_{\lambda}$ and $\varepsilon>0$ sufficiently small. Then there exist $g^{ \pm} \in \mathscr{C}_{0}^{\infty}\left(M_{\tau, \varepsilon}^{ \pm}\right)$such that $f_{\lambda}=R_{\lambda} g^{+}=R_{\lambda} g^{-}$and

$$
f_{\lambda}(x)=\left(N_{\tau, \lambda}^{+} R^{+} g^{-}\right)(x)=\left(N_{\tau, \lambda}^{-} R^{-} g^{+}\right)(x), \quad x \in M_{\tau} .
$$

The functions

$$
f_{\lambda, n}(x):=\sum_{k=1}^{n}\left(\left(-\lambda R^{ \pm} W\right)^{k} R^{ \pm} g^{\mp}\right)(x), \quad x \in M_{\tau},
$$

converge to the restriction of $f_{\lambda}$ to $M_{\tau}$ as $n \rightarrow \infty$, in the topology of $\mathscr{L}^{2}\left(M_{\tau}\right)$

Proof. a) We may find a time slice neighborhood $\Sigma$ of $\Sigma$ such that $K \subset J^{+}(\Sigma) \backslash \Sigma$ or $K \subset J^{-}(\Sigma) \backslash \Sigma$. Then the unique solution $f_{0}[u]$ of $D$ with Cauchy data $u$ on $\Sigma$ can be written as $f_{0}[u]=R g$, where $g \in \mathscr{C}_{0}^{\infty}(\Sigma)$. Let $f_{\lambda}:=R_{\lambda} g$. Then $f_{\lambda} \in \operatorname{Sol}_{\lambda}$, and as $\left.f_{\lambda}\right|_{\Sigma}=\left.f_{0}[u]\right|_{\Sigma}, f_{\lambda}$ has Cauchy data $u$ on $\Sigma$. Uniqueness of this solution follows as in the proof of Proposition $2.13 a$ ).

b) This is immediate from our construction of the fundamental solutions $R_{\lambda}^{ \pm}$.

The results presented so far show a strong similarity to the well-known results in the solution theory of normally hyperbolic differential operators. We next show that despite this similarity, the Cauchy problem is in general ill-posed in the present context. This will be done with two examples.

Example 2.15 (Cauchy problem with no solution). Let $D=\square$ be the d'Alembert operator, $\Sigma$ a Cauchy hyperplane, and $W h:=\left\langle w_{1}, h\right\rangle w_{2}$ with $w_{1}, w_{2} \neq 0$ such that $\operatorname{supp} w_{1} \subset \mathcal{O}_{1}$, supp $w_{2} \subset \mathcal{O}_{2}$ with two spacelike separated double cones $\mathcal{O}_{1}, \mathcal{O}_{2}$ over $\Sigma$ (see Figure 2), such that that $R w_{2} \neq 0$. Pick Cauchy data $u$ on $\Sigma$ supported in $\mathcal{O}_{1}$ such that $f_{0}[u]$, the unique solution of $D$ with these Cauchy data, satisfies $\left\langle w_{1}, f_{0}[u]\right\rangle \neq 0$. Then there exists no $f_{\lambda} \in \operatorname{Sol}_{\lambda}$ with Cauchy data $u$.

Proof. We first observe that the assumptions made can easily be satisfied by suitably adjusting $w_{1}, w_{2}$. For a proof by contradiction, assume $f_{\lambda} \in \operatorname{Sol}_{\lambda}$ has Cauchy data $u=\left(u_{0}, u_{1}\right)$ supported only in $\mathcal{O}_{1} \cap \Sigma$. Due to the form of $W h=\left\langle w_{1}, h\right\rangle \cdot w_{2}$ and the fact that $\operatorname{supp} w_{2}$ is disjoint from $\mathcal{O}_{1}$, one observes that $\left(D f_{\lambda}\right)(x)=0$, $x \in \mathcal{O}_{1}$. Hence the restriction of $f_{\lambda}$ to $\mathcal{O}_{1}$ is a solution of $D$, and as $\mathcal{O}_{1}$ is causally convex, this solution is uniquely determined by its Cauchy data $u$ (which are entirely contained in $\mathcal{O}_{1}$ ), i.e. $\left.f_{\lambda}\right|_{\mathcal{O}_{1}}=\left.f_{0}[u]\right|_{\mathcal{O}_{1}}$, where $f_{0}[u]$ is the solution of $D$ on $\mathbb{R}^{n}$ with Cauchy data $u$. 


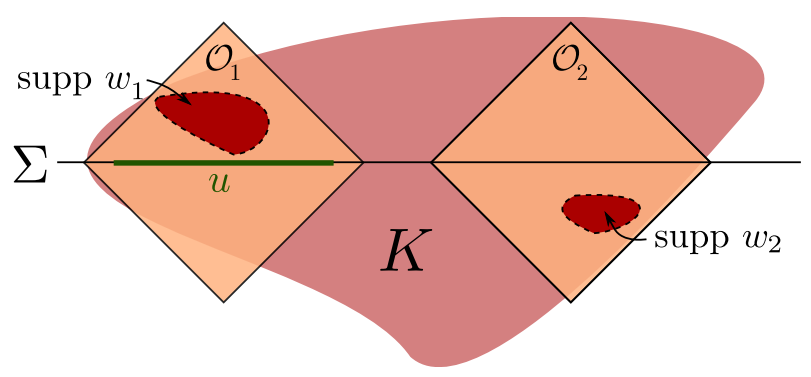

Figure 2. Sketch of the geometric situation of Example 2.15.

We next determine $f_{\lambda}$ on $\mathcal{O}_{2}$. To this end, we use the equation (2.21) with the Cauchy hyperplane $\Sigma$ considered here. Inserting the special form of $W$ and $U^{0}=0$, and taking into account the supports of $w_{1}, w_{2}$, we obtain with $h \in \mathscr{C}_{0}^{\infty}$ and $T_{\lambda}^{ \pm}=R_{\lambda}^{\mp}$ since $D=D^{*}$,

$$
\begin{aligned}
\left\langle h, f_{\lambda}\right\rangle-\lambda\left\{\overline{\left\langle w_{2}, R_{\lambda}^{+} h\right\rangle} \int_{\Sigma_{t}^{+}} d x\left(w_{1}(x), f_{\lambda}(x)\right)+0-0\right. \\
\left.\quad-\left\langle w_{1}, f_{\lambda}\right\rangle \int_{\Sigma_{t}^{-}} d x\left(\left(R_{\lambda}^{-} h\right)(x), w_{2}(x)\right)\right\} \\
=\left\langle h, f_{\lambda}\right\rangle-\lambda\left\{\left\langle R_{\lambda}^{+} h, w_{2}\right\rangle\left\langle w_{1}, f_{\lambda}\right\rangle-\left\langle w_{1}, f_{\lambda}\right\rangle\left\langle R_{\lambda}^{-} h, w_{2}\right\rangle\right\} \\
=\left\langle h, f_{\lambda}\right\rangle+\lambda\left\langle h, R_{\lambda} w_{2}\right\rangle\left\langle w_{1}, f_{\lambda}\right\rangle \\
=\int_{\Sigma_{t}}\left\{\left(\left(R_{\lambda} h\right)_{t}, u_{1}\right)-\left(\left(\partial_{0} R_{\lambda} h\right)_{t}, u_{0}\right)\right\} .
\end{aligned}
$$

For $h \in \mathscr{C}^{\infty}\left(\mathcal{O}_{2}\right)$, we have $W R^{ \pm} h=0$ because supp $w_{1}$ is spacelike to $\mathcal{O}_{2}$, and thus $R_{\lambda} h=R h$. (This holds in particular for $h=w_{2}$.) Hence, by the assumption on the Cauchy data of $f_{\lambda}$, the integral over $\Sigma=\Sigma_{t}$ above vanishes for all such $h$. In view of the above equality, we then have $0=\left\langle h, f_{\lambda}+\lambda\left\langle w_{1}, f_{\lambda}\right\rangle R_{\lambda} w_{2}\right\rangle$, and since $h \in \mathscr{C}^{\infty}\left(\mathcal{O}_{2}\right)$ was arbitrary,

$$
f_{\lambda}(x)=-\lambda\left\langle w_{1}, f_{\lambda}\right\rangle\left(R_{\lambda} w_{2}\right)(x)=-\lambda\left\langle w_{1}, f_{0}[u]\right\rangle\left(R w_{2}\right)(x), \quad x \in \mathcal{O}_{2} .
$$

Thus the Cauchy data of $f_{\lambda}$ and $R w_{2}$ on $\mathcal{O}_{2} \cap \Sigma$ differ only by the (non-zero) factor $-\lambda\left\langle w_{1}, f_{0}[u]\right\rangle$. But by assumption, these Cauchy data are zero. Furthermore, the Cauchy data of $R w_{2}$ on $\Sigma$ can have only support in $\mathcal{O}_{2}$ since supp $w_{2} \subset \mathcal{O}_{2}$, and thus we conclude that $R w_{2}$, as a solution of $D$, must vanish identically. This is a contradiction.

Example 2.16 (Cauchy Problem with non-unique solution). Let again $W f=$ $\left\langle w_{1}, f\right\rangle w_{2}$, with $w_{1}, w_{2} \in \mathscr{C}_{0}^{\infty}$ with spacelike separated supports, and a Cauchy 
hyperplane $\Sigma$ such that supp $w_{2} \subset \Sigma^{-}$. Denoting the Cauchy data of $R w_{2}$ on $\Sigma$ by $u$, let $f_{\lambda}:=f_{0}[u]-R^{+} w_{2}$. Then $w_{1}$ and $\lambda \neq 0$ can be chosen in such a way that the $R_{\lambda}^{ \pm}$exist, $f_{\lambda}$ is a non-zero solution of $D_{\lambda}$, and $f_{\lambda}$ has zero Cauchy data on $\Sigma$.

Proof. We first note that by construction, both $f_{0}[u]$ and $R^{+} w_{2}$ have Cauchy data $u$ on $\Sigma$, and thus $f_{\lambda}$ has zero Cauchy data. As the support of $f_{0}[u]$ extends to infinitely late times, whereas $\operatorname{supp}\left(R^{+} w_{2}\right) \subset J^{+}\left(\operatorname{supp} w_{2}\right)$ extends only to the future, $f_{\lambda}$ is non-zero. We calculate

$$
\begin{aligned}
D_{\lambda} f_{\lambda} & =D f_{0}[u]-D R^{+} w_{2}+\lambda\left\langle w_{1}, f_{0}[u]-R^{+} w_{2}\right\rangle w_{2} \\
& =\left(-1+\lambda\left\langle w_{1}, f_{0}[u]-R^{+} w_{2}\right\rangle\right) \cdot w_{2} \\
& =\left(-1+\lambda\left\langle w_{1}, f_{0}[u]\right\rangle\right) \cdot w_{2},
\end{aligned}
$$

and have to make sure that $\left\langle w_{1}, f_{0}[u]\right\rangle \neq 0$, so that $D_{\lambda} f_{\lambda}=0$. This can be done by adjusting $w_{1}$ suitably. Then $f_{\lambda}$ is a non-zero solution of $D_{\lambda}$ for $\lambda=1 /\left\langle w_{1}, f_{0}[u]\right\rangle \neq 0$.

Moreover, the fundamental solutions $R_{\lambda}^{ \pm}$exist for this value of $\lambda$. In fact, since $\operatorname{supp} w_{1}$ lies spacelike to supp $w_{2}$, we have $W R^{ \pm} W=0$, so that the Neumann series (2.7) terminate, and thus converge for all $\lambda \in \mathbb{C}$.

As these examples demonstrate, the Cauchy problem for $D_{\lambda}$ is in general illposed for Cauchy hyperplanes $\Sigma$ such that $K \not \subset \Sigma^{+}$and $K \not \subset \Sigma^{-}$- both existence and uniqueness of solutions can fail. What can however be analyzed is the scattering of free solutions of $D$ at the perturbation $W$, closely related to the relative Cauchy evolution $[3,22,32]$. This is the topic of the next section.

2.3. Scattering. We have seen before that any solution $f_{\lambda} \in \operatorname{Sol}_{\lambda}$ restricts to free solutions $f_{0}^{ \pm} \in \operatorname{Sol}_{0}$ in the future $\Sigma_{\tau_{+}}^{+}$and past $\Sigma_{\tau_{-}}^{-}$of the perturbation $W$. In general, it will not be possible to prescribe solutions $f_{0}^{+}$and $f_{0}^{-}$of $D$ such that $f_{\lambda}(x)=f_{0}^{+}(x)$ for $x_{0}>\tau_{+}$and $f_{\lambda}(x)=f_{0}^{-}(x)$ for $x_{0}<\tau_{-}$, as $f_{0}^{+}$is uniquely determined by $f_{0}^{-}$and vice versa. The relation between these "incoming free asymptotics" to "outgoing free asymptotics" - where "free" refers here to the unperturbed differential operator $D$ - is nothing but the scattering at the non-local potential $W$, which we are going to analyze next. We first define two Møller type operators

$$
\begin{aligned}
& \Omega_{\lambda, \pm}: \operatorname{Sol}_{\lambda} \rightarrow \operatorname{Sol}_{0}, \\
& \Omega_{\lambda, \pm}: R_{\lambda} g \mapsto R g, \quad g \in \mathscr{C}_{0}^{\infty}\left(\Sigma_{\tau_{ \pm}}^{ \pm}\right) .
\end{aligned}
$$

\section{Proposition 2.17 (Møller operators).}

a) The Møller operators $\Omega_{\lambda, \pm}$ are well-defined linear bijections. 
b) Given a solution $f \in \operatorname{Sol}_{\lambda}$, we have

$$
\left.f\right|_{\Sigma_{\tau_{ \pm}}^{ \pm}}=\left.\left(\Omega_{\lambda, \pm} f\right)\right|_{\Sigma_{\tau_{ \pm}}^{ \pm}}
$$

c) The Moller operators $\Omega_{\lambda, \pm}$ intertwine the sesquilinear forms $\rho_{0}$ and $\rho_{\lambda}$ defined in (2.26), i.e.

$$
\rho_{0}\left(\Omega_{\lambda, \pm} f_{\lambda}, \Omega_{\lambda, \pm} g_{\lambda}\right)=\rho_{\lambda}\left(f_{\lambda}, g_{\lambda}\right), \quad f_{\lambda}, g_{\lambda} \in \operatorname{Sol}_{\lambda}
$$

Proof. a) Let $g \in \mathscr{C}_{0}^{\infty}\left(\Sigma_{\tau_{ \pm}}^{ \pm}\right)$and assume that $R_{\lambda} g=0$. Then in particular the restriction $\left.\left(R_{\lambda} g\right)\right|_{\Sigma_{\tau_{ \pm}}^{ \pm}}$vanishes. But on $\Sigma_{\tau_{ \pm}}^{ \pm}$, we have $0=\left.\left(R_{\lambda} g\right)\right|_{\Sigma_{\tau_{ \pm}}^{ \pm}}=$ $\left.(R g)\right|_{\Sigma_{\tau_{ \pm}}^{ \pm}}$, and as $R g$ is a solution of $D$, this implies that $R g=0$ on all of $\mathbb{R}^{n}$. Hence the assignment (2.32) is well-defined and injective.

By Proposition $2.13 a$ ), $\operatorname{Sol}_{\lambda}=R_{\lambda} \mathscr{C}_{0}^{\infty}\left(\Sigma_{\tau_{+}}^{ \pm}\right)$. Thus (2.32) defines in fact a linear mapping from $\mathrm{Sol}_{\lambda}$ to $\mathrm{Sol}_{0}$, and also surjectivity is immediate from (2.32) and Proposition $2.13 a$ ).

b) Let $f=R_{\lambda} g \in \operatorname{Sol}_{\lambda}, g \in \mathscr{C}_{0}^{\infty}\left(\Sigma_{\tau_{ \pm}}^{ \pm}\right)$. Then $\left.f\right|_{\Sigma_{\tau_{ \pm}}^{ \pm}}=\left.R g\right|_{\Sigma_{\tau_{ \pm}}^{ \pm}}$. This is the same as (2.33).

c) Let $f_{\lambda}, g_{\lambda} \in \operatorname{Sol}_{\lambda}$. By Proposition $\left.2.13 a\right)$, we find $f^{ \pm}, g^{ \pm} \in \mathscr{C}_{0}^{\infty}\left(\Sigma_{\tau_{ \pm}}^{ \pm}\right)$such that $f_{\lambda}=R_{\lambda} f^{+}=R_{\lambda} f^{-}, g_{\lambda}=R_{\lambda} g^{+}=R_{\lambda} g^{-}$and therefore, $\Omega_{\lambda, \pm} f_{\lambda}=R f^{ \pm}$, $\Omega_{\lambda, \pm} g_{\lambda}=R g^{ \pm}$. Thus, the left and right hand sides of (2.34) can be written as

$$
\begin{aligned}
\rho_{0}\left(\Omega_{\lambda, \pm} f_{\lambda}, \Omega_{\lambda, \pm} g_{\lambda}\right) & =\rho_{0}\left(R f^{ \pm}, R g^{ \pm}\right)=\left\langle f^{ \pm}, R g^{ \pm}\right\rangle, \\
\rho_{\lambda}\left(f_{\lambda}, g_{\lambda}\right) & =\rho_{\lambda}\left(R_{\lambda} f^{ \pm}, R_{\lambda} g^{ \pm}\right)=\left\langle f^{ \pm}, R_{\lambda} g^{ \pm}\right\rangle .
\end{aligned}
$$

But as in part b), we have $\left.\left(R_{\lambda}^{ \pm} g^{ \pm}\right)\right|_{\Sigma_{\tau_{ \pm}}^{ \pm}}=\left.\left(R^{ \pm} g^{ \pm}\right)\right|_{\Sigma_{\tau_{ \pm}}^{ \pm}}$, and consequently $\left\langle f^{ \pm}, R_{\lambda} g^{ \pm}\right\rangle=\left\langle f^{ \pm}, R g^{ \pm}\right\rangle$.

We can now introduce the scattering operator

$$
S_{\lambda}:=\Omega_{\lambda,+}\left(\Omega_{\lambda,-}\right)^{-1}: \operatorname{Sol}_{0} \rightarrow \text { Sol }_{0},
$$

which maps the incoming asymptotics $\Omega_{\lambda,-} f$ of a solution $f \in \operatorname{Sol}_{\lambda}$ to its outgoing asymptotics $\Omega_{\lambda,+} f$, and thus describes the scattering by the potential term $\lambda W$ [3].

\section{Theorem 2.18 (Scattering operator).}

a) The scattering operator $S_{\lambda}: \mathrm{Sol}_{0} \rightarrow \mathrm{Sol}_{0}(2.35)$ is a linear bijection.

b) $S_{\lambda}$ preserves the sesquilinear form $\rho_{0}(2.26)$, i.e.

$$
\rho_{0}\left(S_{\lambda} f, S_{\lambda} g\right)=\rho_{0}(f, g), \quad f, g \in \operatorname{Sol}_{0} .
$$


c) Explicitly, $S_{\lambda}$ is given by

$$
S_{\lambda}=1+\lambda R W N_{\tau, \lambda}^{+}=1+R W \sum_{k=0}^{\infty} \lambda^{k+1}\left(-R^{+} W\right)^{k} .
$$

The sum converges in the norm of bounded operators on $\mathscr{L}^{2}\left(M_{\tau}\right)$.

d) For any $f_{0} \in \mathrm{Sol}_{0}$,

$$
\lambda \longmapsto S_{\lambda} f_{0}
$$

is analytic in the topology of $\mathscr{C}^{\infty}$ on a finite disc around $\lambda=0$. In particular, $f_{0} \in \mathrm{Sol}_{0}$,

$$
\left.\frac{d\left(S_{\lambda} f_{0}\right)}{d \lambda}\right|_{\lambda=0}=R W f_{0} .
$$

Proof. Part $a$ ) is clear from Proposition $2.17 a$ ). For $b$ ), we use Proposition $2.17 c$ ) and invertibility of the Møller operators to find, $f, g \in \mathrm{Sol}_{0}$,

$$
\begin{aligned}
\rho_{0}\left(S_{\lambda} f, S_{\lambda} g\right) & =\rho_{0}\left(\Omega_{\lambda,+}\left(\Omega_{\lambda,-}\right)^{-1} f, \Omega_{\lambda,+}\left(\Omega_{\lambda,-}\right)^{-1} g\right) \\
& =\rho_{\lambda}\left(\left(\Omega_{\lambda,-}\right)^{-1} f,\left(\Omega_{\lambda,-}\right)^{-1} g\right) \\
& =\rho_{0}(f, g) .
\end{aligned}
$$

c) Given a solution $f_{0} \in \mathrm{Sol}_{0}$,

$$
\psi:=W N_{\tau, \lambda}^{+} f_{0}=W \sum_{k=0}^{\infty}\left(-\lambda R_{\tau}^{+} W\right)^{k} f_{0}
$$

is well-defined because each term is restricted to $K \subset M_{\tau}$ by the action of $W$. Since $W$ is smoothing, we have $\psi \in \mathscr{C}_{0}^{\infty}(K)$. This implies that with $f_{0}$, also $f_{0}+\lambda R \psi$ is a solution of $D$, i.e $T_{\lambda}:=1+\lambda R W N_{\tau, \lambda}^{+}$is a well-defined linear map $T_{\lambda}: \mathrm{Sol}_{0} \rightarrow \mathrm{Sol}_{0}$.

Any solution of $D$ is uniquely determined by its restriction to $M_{\tau}$. To prove $T_{\lambda}=S_{\lambda}$, it is therefore sufficient to prove that the restrictions of $S_{\lambda} f_{0}$ and $T_{\lambda} f_{0}$ to $M_{\tau}$ coincide.

To do so, we consider a solution $f_{\lambda} \in \operatorname{Sol}_{\lambda}$. Then we find $\varepsilon>0$ and $g^{ \pm} \in$ $\mathscr{C}_{0}^{\infty}\left(M_{\tau, \varepsilon}^{ \pm}\right)$such that $f_{\lambda}=R_{\lambda} g^{+}=R_{\lambda} g^{-}$and $f_{0}:=R g^{-}=\Omega_{\lambda,-} f_{\lambda}$ as well as $S_{\lambda} f_{0}=\Omega_{\lambda,+} f_{\lambda}=R g^{+}$, by definition of $\Omega_{\lambda, \pm}$ and $S_{\lambda}$. As every solution $f_{0} \in \mathrm{Sol}_{0}$ arises in this way, what is left to prove is

$$
\left.\left(R g^{+}\right)\right|_{M_{\tau}}=\left.\left(T_{\lambda} R g^{-}\right)\right|_{M_{\tau}}
$$


To this end, we compute

$$
\begin{aligned}
\left.f_{\lambda}\right|_{M_{\tau}} & =R_{\tau, \lambda} g^{+} \\
& =R_{\tau, \lambda}^{-} g^{+}-R_{\tau, \lambda}^{+} g^{+} \\
& =N_{\tau, \lambda}^{-} R_{\tau}^{-} g^{+}-R_{\tau}^{+} g^{+} \\
& =\left(1-\lambda N_{\tau, \lambda}^{-} R_{\tau}^{-} W\right) R_{\tau}^{-} g^{+}-R_{\tau}^{+} g^{+} \\
& =R_{\tau} g^{+}-\lambda N_{\tau, \lambda}^{-} R_{\tau}^{-} W R_{\tau}^{-} g^{+} \\
& =R_{\tau} g^{+}-\lambda N_{\tau, \lambda}^{-} R_{\tau}^{-} W R_{\tau} g^{+} \\
& =\left(1-\lambda N_{\tau, \lambda}^{-} R_{\tau}^{-} W\right) R_{\tau} g^{+} \\
& =N_{\tau, \lambda}^{-} R_{\tau} g^{+},
\end{aligned}
$$

where we have used the definition of $R_{\tau, \lambda}^{ \pm}$, equation (2.10), and the fact that because of the supports of $g^{+}$and $W$, we have $W R_{\tau}^{-} g^{+}=W R_{\tau} g^{+}$. In complete analogy, one computes $\left.f_{\lambda}\right|_{M_{\tau}}=N_{\tau, \lambda}^{+} R_{\tau} g^{-}$.

We thus have $\left.\left(R g^{+}\right)\right|_{M_{\tau}}=R_{\tau} g^{+}=\left(N_{\tau, \lambda}^{-}\right)^{-1}\left(\left.f_{\lambda}\right|_{M_{\tau}}\right)=\left(N_{\tau, \lambda}^{-}\right)^{-1} N_{\tau, \lambda}^{+} R_{\tau} g^{-}$. Using the equation $(1+X)(1+Y)^{-1}=1-(Y-X)(1+Y)^{-1}$, valid for operators $X, Y$ with $\|X\|,\|Y\|<1$, we find

$$
\begin{aligned}
\left.\left(R g^{+}\right)\right|_{M_{\tau}} & =\left(N_{\tau, \lambda}^{-}\right)^{-1} N_{\tau, \lambda}^{+} R_{\tau} g^{-} \\
& =\left(1+\lambda R_{\tau}^{-} W\right)\left(1+\lambda R_{\tau}^{+} W\right)^{-1} R_{\tau} g^{-} \\
& =\left(1+\lambda R_{\tau} W N_{\tau, \lambda}^{+}\right) R_{\tau} g^{-} \\
& =\left.\left(T_{\lambda} R g^{-}\right)\right|_{M_{\tau}} .
\end{aligned}
$$

This shows (2.40) and thus $S_{\lambda}=T_{\lambda}$. The second equality in (2.37) follows by inserting the definition of $N_{\tau, \lambda}^{+}$.

d) For $f_{0} \in \operatorname{Sol}_{0}$, we have $W f_{0} \in \mathscr{C}_{0}^{\infty}(K) \subset \mathscr{L}^{2}\left(M_{\tau}\right)$. As the Neumann series $N_{\tau, \lambda}^{+}$converges in the norm of $\mathcal{B}\left(\mathscr{L}^{2}\left(M_{\tau}\right)\right)$, the function

$$
\lambda \longmapsto N_{\tau, \lambda}^{+} f_{0}=f_{0}-\lambda N_{\tau, \lambda}^{+} R_{\tau}^{+} W f_{0} \in \mathscr{L}^{2}\left(M_{\tau}\right)
$$

is analytic (in the norm topology of $\mathscr{L}^{2}\left(M_{\tau}\right)$ ) for sufficiently small $|\lambda|$. But $W: \mathscr{L}^{2}\left(M_{\tau}\right) \rightarrow \mathscr{C}_{0}^{\infty}$ and $R: \mathscr{C}_{0}^{\infty} \rightarrow \mathscr{C}^{\infty}$ are linear and continuous. Hence $\lambda \mapsto f_{0}+\lambda R W N_{\tau, \lambda}^{+} f_{0} \in \mathscr{C}^{\infty}$ is analytic in the topology of $\mathscr{C}^{\infty}$. According to $c$ ), this function coincides with $\lambda \mapsto S_{\lambda} f_{0}$.

In view of this analyticity, we can differentiate under the sum in (2.37) and immediately obtain $\left.\partial_{\lambda} S_{\lambda} f_{0}\right|_{\lambda=0}=R W f_{0}, f_{0} \in \operatorname{Sol}_{0}$. 


\section{Perturbations by star products}

In this section we discuss two examples of perturbations $W$ which are not $\mathscr{C}_{0}^{\infty}$-kernel operators, but rather limits thereof. These examples arise in the context of (classical) field theory of noncommutative spaces, where one seeks to describe the dynamics in the presence of a noncommutatively coupled potential. For the case of a Dirac operator and a star product which is commutative in time, such an analysis was carried out in [13]. Here we can generalize to the case of noncommutative time.

We will present two examples, each of which violates one the important properties of $\mathscr{C}_{0}^{\infty}$-kernel operators, namely either the smoothness or the compact support of the kernel. We will not fully analyze these perturbations here, but rather show how they fit in the framework described previously as limits of $\mathscr{C}_{0}^{\infty}$-kernel operators, and that the important scattering derivation $\left.\partial_{\lambda} S_{\lambda}\right|_{\lambda=0}$ still exists here.

The basic structure we will be concerned with is that of Rieffel's product [37]. Thus the main ingredient is an action $\alpha$ of $\mathbb{R}^{n}$. In our context, $\alpha$ will act on various function spaces (for simplicity, we here take $N=1$, i.e. consider scalar functions) by pullback of an $\mathbb{R}^{n}$-action $\tau$ on $\mathbb{R}^{n}$, i.e. by $\left(\alpha_{z} f\right)(x)=f\left(\tau_{z}(x)\right)$. Picking also an antisymmetric, invertible, real $(n \times n)$-matrix as deformation parameter, we consider products of the form

$$
w \star f:=\int_{\mathbb{R}^{n}} d p \int_{\mathbb{R}^{n}} d z e^{2 \pi i(p, z)} \alpha_{\theta p} w \cdot \alpha_{z} f .
$$

For our purposes, we will always take $w \in \mathscr{C}_{0}^{\infty}$, and $f$ will be a smooth function on $\mathbb{R}^{n}$ with falloff properties depending on the choice of $\tau$.

The best known example is to take $\tau_{z}(x)=x+z$ and $f \in \mathscr{S}\left(\mathbb{R}^{n}\right)$ (Schwartz space). In this case $\star$ coincides with the Moyal product, and we have a continuous associative but noncommutative product $\star$.

Another class of examples has been discussed in [34], see also [14, 31] for earlier related work. There the idea is to take $\tau$ of such a form that it leaves a compact set $K \subset \mathbb{R}^{n}$ invariant. In more detail, such an action $\tau$ can for example be constructed as follows [31,34]. Let $\gamma:(-1,1) \rightarrow \mathbb{R}$ be a diffeomorphism, and define, $x=\left(x_{1}, \ldots, x_{n}\right), z=\left(z_{1}, \ldots, z_{n}\right) \in \mathbb{R}^{n}$,

$$
\tau_{z}(x)_{k}:=\left\{\begin{array}{ll}
\gamma^{-1}\left(\gamma\left(x_{k}\right)+z_{k}\right) & \left|x_{k}\right|<1 \\
x_{k} & \left|x_{k}\right| \geq 1
\end{array} .\right.
$$

Clearly $\tau$ is an $\mathbb{R}^{n}$-action, and $K:=[-1,1]^{n}$ is invariant under $\tau$. When $\gamma$ is appropriately chosen, $\tau$ is also smooth and polynomially bounded. We recall from [34, Sect. 5] that this can be achieved by choosing $\gamma$ such that $\gamma$ is antisymmetric, $\gamma\left(x_{k}\right)=\exp \left(\frac{1}{1-x_{k}}\right)$ for $x_{k}>\frac{1}{2}$, and $\gamma^{\prime}\left(x_{k}\right) \geq \gamma^{\prime}(0)>0$. In this case, one can take $f \in \mathscr{C}^{\infty}$, and again obtain a continuous associative but noncommutative product $\star$. 
In both these situations, the one of the canonical translations $\tau$ and the the one just discussed, the integral has to understood as an oscillatory integral taking values in $\mathscr{S}\left(\mathbb{R}^{n}\right)$ and $\mathscr{C}^{\infty}\left(\mathbb{R}^{n}\right)$, respectively. Concretely, it can always be calculated according to

$$
(w \star f)(x)=\lim _{\varepsilon \rightarrow 0} \int_{\mathbb{R}^{n}} d p \int_{\mathbb{R}^{n}} d z e^{2 \pi i(p, z)} \chi(\varepsilon p) \chi(\varepsilon z) w\left(\tau_{\theta p}(x)\right) f\left(\tau_{z}(x)\right),
$$

where $\chi \in \mathscr{C}_{0}^{\infty}\left(\mathbb{R}^{n}\right)$ is a cutoff function, equal to 1 on an open neighborhood of 0 , and $w \star f$ is independent of the choice of $\chi$.

In the following, we will consider for $w \in \mathscr{C}_{0}^{\infty}$ the perturbation term

$$
W f:=w \star f,
$$

and denote by $W_{\varepsilon}$ the integral operator in (3.2), $\varepsilon>0$. When the two cases need to be distinguished, we will also write $W_{(\varepsilon)}^{M}$ and $W_{(\varepsilon)}^{K}$, respectively. To simplify matters, we will also require that the support of $w$ is contained in the interior of $K$ in the case of $W^{K}$.

Lemma 3.1 (Properties of star product kernels). Let $w \in \mathscr{C}_{0}^{\infty}$ and $W=W^{M}$ or $W=W^{K}$ defined as in (3.3).

a) Let $\varepsilon>0$. Then $W_{\varepsilon}$ is a $\mathscr{C}_{0}^{\infty}$-kernel operator.

b) The integral kernel of $W^{M}$ is smooth, but not of compact support.

c) The integral kernel of $W^{K}$ is of compact support (in K), but not smooth.

Proof. We first consider the Moyal product. Then we have

$$
\begin{aligned}
\left(W_{\varepsilon}^{M} f\right)(x) & =\int_{\mathbb{R}^{n}} d p \int_{\mathbb{R}^{n}} d z e^{2 \pi i(p, z)} \chi(\varepsilon p) \chi(\varepsilon z) w(x+\theta p) f(x+z) \\
& =\int_{\mathbb{R}^{n}} d y\left(\int_{\mathbb{R}^{n}} d p e^{2 \pi i(p,(y-x))} \chi(\varepsilon p) \chi(\varepsilon(y-x)) w(x+\theta p)\right) f(y) .
\end{aligned}
$$

Because both $w$ and $\chi$ have compact support, $\chi(\varepsilon p) w(x+\theta p)$ vanishes for all $p$ if $|x|$ is large enough. Furthermore, $\chi(\varepsilon(y-x))=0$ if $|x-y|$ is large enough. Thus $W_{\varepsilon}^{M}$ has a kernel of compact support. The smoothness of this kernel follows from well-known statements on the Fourier transform, i.e. we have shown $a$ ) for the Moyal product.

For fixed $x$, the limit $\varepsilon \rightarrow 0$ can be taken under the integral, and after a change of variables one finds

$$
\left(W^{M} f\right)(x)=\frac{1}{(2 \pi)^{n / 2}|\operatorname{det} \theta|} \int d y e^{i x \cdot \theta^{-1} y} \widetilde{w}\left(\theta^{-1}(y-x)\right) f(y),
$$


where $\widetilde{w}$ denotes the Fourier transform of $w$. From this formula it is obvious that the kernel of $W^{M}$ is smooth and not compactly supported, i.e. we have shown $b$ ).

Now we consider the locally noncommutative product, and observe the following two properties of $w \star f$ : First, if $x \notin K$, then also $\tau_{\theta p}(x) \notin K$ for all $p \in \mathbb{R}^{n}$ since $K$ is invariant and $\tau$ is an action. In view of supp $w \subset K$, we then have $w\left(\tau_{\theta p}(x)\right)=0$ for all $p$, and hence $(w \star f)(x)=0$ for $x \notin K$. Second, if supp $f \cap K=\emptyset$, and $x \in K$, then $f\left(\tau_{z}(x)\right)=0$ for all $z \in \mathbb{R}^{n}$, and thus again $(w \star f)(x)=0$. These remarks apply to both $W^{K}$ and $W_{\varepsilon}^{K}$ and show that these operators have kernels supported in $K \times K$.

Explicitly, we find after a change of variables, $x \notin K$,

$$
\begin{aligned}
&\left(W_{\varepsilon}^{K} f\right)(x)=\int_{\mathbb{R}^{n}} d p \int_{\mathbb{R}^{n}} d z e^{2 \pi i(p, z)} \chi(\varepsilon p) \chi(\varepsilon z) w\left(\tau_{\theta p}(x)\right) f\left(\tau_{z}(x)\right) \\
&=\int_{K} \frac{d y \gamma^{\prime}(y)}{|\operatorname{det} \theta|} f(y) \int_{\mathbb{R}^{n}} d p e^{2 \pi i\left(\gamma(x), \theta^{-1} \gamma(y)\right)} e^{2 \pi i(\theta p,(\gamma(y)-\gamma(x))} \\
& \chi(\varepsilon p) \chi(\varepsilon \gamma(y)-\varepsilon \gamma(x)) w\left(\tau_{\theta p}(x)\right),
\end{aligned}
$$

where we used the shorthand notations $\gamma(x):=\left(\gamma\left(x_{1}\right), \ldots, \gamma\left(x_{n}\right)\right)$ and $\gamma^{\prime}(x):=$ $\left(\gamma^{\prime}\left(x_{1}\right), \ldots, \gamma^{\prime}\left(x_{n}\right)\right)$. If the distance of $x$ to the boundary of $K$ is smaller than some minimal distance $d$ (depending on $\varepsilon, \theta$, and the supports of $\chi, w$ ), then $\chi(\varepsilon p) w\left(\tau_{\theta p}(x)\right)=0$ for all $p$ by the support properties of $\chi$ and $w$. Furthermore, if the distance $\varepsilon|\gamma(y)-\gamma(x)|$ is large enough, then $\chi(\varepsilon \gamma(y)-\varepsilon \gamma(x))=0$. Since $\gamma$ diverges as $x$ or $y$ approach the boundary of $K$, this implies that the integral over $p$ vanishes for $(x, y)$ outside some compact set properly contained in the interior of $K$. Thus $W_{\varepsilon}^{K}$ is a $\mathscr{C}_{0}^{\infty}$-kernel operator.

However, the kernel of $W^{K}$ is not smooth. In fact, by a calculation analogous to the one for the Moyal product, one can compute that $W^{K}$ has the integral kernel

$$
\begin{aligned}
\left(W^{K} f\right)(x) & =\frac{1}{(2 \pi)^{n / 2}|\operatorname{det} \theta|} \int d y k(x, y) f(y), \\
k(x, y) & :=\left\{\begin{array}{ll}
\gamma^{\prime}(y) e^{i \gamma(x) \cdot \theta^{-1} \gamma(y)} \widetilde{\varphi}\left(\theta^{-1}(\gamma(y)-\gamma(x))\right) & x, y \in K \\
0 & x \notin K \text { or } y \notin K
\end{array},\right.
\end{aligned}
$$

where $\varphi:=w \circ \gamma^{-1}$ and the tilde denotes a Fourier transform. From this formula, one sees that $k$ is discontinuous at the boundary of $K$, for example by noting that $k(x, x)=\gamma^{\prime}(x)$ diverges as $x$ approaches the boundary of $K$ from the inside.

Both star product operators, $W^{M}$ and $W^{K}$, are thus limits of $\mathscr{C}_{0}^{\infty}$-kernel operators which do not lie in this class themselves. In the case of the locally noncommutative star product, the smoothness of the kernel fails, but each $W_{\varepsilon}^{K}$ has support in the same set $K$. Here it is conceivable that our methods can be generalized in such a way that also $W^{K}$ can be analyzed along the same lines as 
$\mathscr{C}_{0}^{\infty}$-kernel operators, i.e. that its fundamental solutions, scattering operator, etc. can be constructed.

The Moyal star product operator $W^{M}$ differs more drastically from the situation considered so far, as the supports of $W_{\varepsilon}^{M}$ grow infinitely as $\varepsilon \rightarrow 0$ (albeit they have smooth kernels). Here one would need to pass to an asymptotic formulation of the scattering problem.

These matters will be discussed in more detail elsewhere. However, already at the present stage one can show that the derivative of the scattering operator at zero coupling, which is the essential quantity for the connection to Bogoliubov's formula, does exist also for the locally noncommutative multiplier.

Proposition 3.2. Let $W=W^{K}$ be defined as in (3.3). Then $W R_{\tau}^{ \pm}$and $R_{\tau}^{ \pm} W$ extend to bounded operators on $\mathscr{L}^{2}\left(M_{\tau}\right)$.

Proof. The pointwise product $f \mapsto w \cdot f$ is a continuous linear map $\mathscr{C}^{\infty}\left(M_{\tau}\right) \rightarrow$ $\mathscr{C}^{\infty}\left(M_{\tau}\right)$. As $K$ is contained in $M_{\tau}$, the action $\alpha_{x} f:=f \circ \tau_{x}^{K}$ is a smooth polynomially bounded $\mathbb{R}^{n}$-action on $\mathscr{C}^{\infty}\left(M_{\tau}\right)$ [34, Prop. 5.5], and hence, $f \mapsto w \star f$ is also a continuous linear map $\mathscr{C}^{\infty}\left(M_{\tau}\right) \rightarrow \mathscr{C}^{\infty}(K)$ [34, Prop. 4.6]. As $R_{\tau}^{ \pm}: \mathscr{C}_{0}^{\infty}\left(M_{\tau}\right) \rightarrow \mathscr{C}^{\infty}\left(M_{\tau}\right)$ are continuous, we see that $W R_{\tau}^{ \pm}: \mathscr{C}_{0}^{\infty}\left(M_{\tau}\right) \rightarrow$ $\mathscr{C}^{\infty}(K)$ is continuous. But $\mathscr{C}^{\infty}(K)$ embeds continuously in $\mathscr{L}^{2}\left(M_{\tau}\right)$, and $\mathscr{C}_{0}^{\infty}\left(M_{\tau}\right) \subset \mathscr{L}^{2}\left(M_{\tau}\right)$ is dense. Hence $W R_{\tau}^{ \pm}$extends continuously to $\mathscr{L}^{2}\left(M_{\tau}\right) \rightarrow$ $\mathscr{L}^{2}\left(M_{\tau}\right)$. Similarly, $R_{\tau}^{ \pm} W: \mathscr{C}_{0}^{\infty}\left(M_{\tau}\right) \rightarrow \mathscr{C}^{\infty}\left(M_{\tau}\right)$ is continuous, and by the support properties of $R^{ \pm} f$, we can also extend $R_{\tau}^{ \pm}$to $\mathscr{L}^{2}\left(M_{\tau}\right)$ as in Lemma 2.5.

In the case of a Moyal multiplier $W^{M}$, the space of functions on the time slice $M_{\tau}$ is not invariant under $W^{M}$. Rather, one needs to take $\tau \rightarrow \infty$ as $\varepsilon \rightarrow 0$ to guarantee that $W_{\varepsilon}^{M}$ is always supported in $M_{\tau(\varepsilon)}$. Some aspects of this limit have been studied in [8].

\section{CCR and CAR Quantization}

So far we have concentrated on the integro-differential equation $D_{\lambda} f=0$ for classical fields $f$. However, as explained in the Introduction Orange (see also the Outlook in Section 5), most of our motivation comes from analyzing this equation for quantum fields. Thanks to the linearity of $D_{\lambda}$, a quantization of the solution spaces $\mathrm{Sol}_{\lambda}$ is possible in a straightforward manner, as we shall outline in this section. We will distinguish two cases: The case of a symmetric differential operator, which naturally leads to CCR quantization, and the case of a Dirac operator, which naturally leads to CAR quantization. For (pre-)normally differential operators $D$ without the non-local perturbation $W$, such an analysis has been carried out in [4], and for Dirac operators with a perturbation which is local in time, see [13]. 
We begin with the CCR case and assume that $D$ is a symmetric differential operator with symmetric perturbation $W=W^{*}$ (and real coupling $\lambda$ ). We also introduce a conjugation $C$ on $\mathbb{C}^{N}$, i.e. an antiunitary involution $C: \mathbb{C}^{N} \rightarrow \mathbb{C}^{N}$. By pointwise action, $C$ operates also on all function spaces appearing here, and will be denoted by the same symbol $C$ everywhere. Note that on $\mathscr{L}^{2}$, the so defined conjugation is antiunitary. To single out a real space of solutions, we assume that

$$
D C f=C D f, \quad W C f=C W f, \quad f \in \mathscr{C}_{0}^{\infty} .
$$

An example for this situation is given by the Klein-Gordon operator $D=\square+V(x)$ and an integral operator $W_{w}(2.5)$, where $V \in C^{\infty}\left(\mathbb{R}^{n}, \mathbb{C}^{N \times N}\right), w \in C_{0}^{\infty}\left(\mathbb{R}^{n} \times \mathbb{R}^{n}\right.$, $\mathbb{C}^{N \times N}$ ) are potentials which take only real values in some basis of $\mathbb{C}^{N}$, and $C$ is complex conjugation in that basis.

For real $\lambda$, then also $D_{\lambda}=D+\lambda W$ and $C$ commute, and we define

$$
\operatorname{Sol}_{\lambda, C}:=\left\{f \in \operatorname{Sol}_{\lambda}: C f=f\right\} .
$$

\section{Proposition 4.1 (Symplectic structure of solution spaces).}

a) Under the assumptions made, the sesquilinear form $\rho_{\lambda}$ (2.26) restricts to a real-valued real bilinear non-degenerate symplectic form $\sigma_{\lambda}: \operatorname{Sol}_{\lambda, C} \times$ $\mathrm{Sol}_{\lambda, C} \rightarrow \mathbb{R}$.

b) The Moller operators $\Omega_{\lambda, \pm}$ and the scattering operator $S_{\lambda}$ restrict to symplectomorphisms $\Omega_{\lambda, \pm}:\left(\operatorname{Sol}_{\lambda, C}, \sigma_{\lambda}\right) \rightarrow\left(\operatorname{Sol}_{0, C}, \sigma_{0}\right)$ and $S_{\lambda}:\left(\operatorname{Sol}_{0, C}, \sigma_{0}\right) \rightarrow$ $\left(\mathrm{Sol}_{0, C}, \sigma_{0}\right)$, respectively.

Proof. a) It is clear that $\sigma_{\lambda}$ is real bilinear, and using a decomposition into real and imaginary parts w.r.t. $C$, one also sees that $\sigma_{\lambda}$ inherits non-degenerateness from $\rho_{\lambda}$.

As the conjugation $C$ preserves supports, we have $\operatorname{supp}\left(C R_{\lambda}^{ \pm} C f\right)=$ $\operatorname{supp}\left(R_{\lambda}^{ \pm} C f\right) \subset J^{ \pm}(\operatorname{supp} f) \cup J^{ \pm}(K), f \in \mathscr{C}_{0}^{\infty}$. Furthermore, $C R_{\lambda}^{ \pm} C D f=$ $C R_{\lambda}^{ \pm} D C f=C^{2} f=f$ and similarly, $D C R_{\lambda}^{ \pm} C f=f$. In view of the uniqueness of the fundamental advanced and retarded solutions (Proposition 2.12), we conclude $C R_{\lambda}^{ \pm} C=R_{\lambda}^{ \pm}$. This implies that $\sigma_{\lambda}$ is real-valued: For any $f, g \in \mathscr{C}_{0}^{\infty}, C f=f$, $C g=g$, we have

$$
\begin{aligned}
\sigma_{\lambda}\left(R_{\lambda} f, R_{\lambda} g\right)=\left\langle f, R_{\lambda} g\right\rangle & =\left\langle C f, R_{\lambda} C g\right\rangle \\
& =\overline{\left\langle f, C R_{\lambda} C g\right\rangle}=\overline{\left\langle f, R_{\lambda} g\right\rangle}=\overline{\sigma_{\lambda}\left(R_{\lambda} f, R_{\lambda} g\right)} .
\end{aligned}
$$

Antisymmetry of $\sigma_{\lambda}$ follows now from (2.27).

b) The definition (2.32) of $\Omega_{\lambda, \pm}$ and the fact that $C R_{\lambda} C=R_{\lambda}, C R C=R$ implies that $\Omega_{\lambda, \pm}$ maps $\operatorname{Sol}_{\lambda, C}$ onto $\operatorname{Sol}_{0, C}$. The fact that these operators are symplectic follows from Proposition $2.17 \mathrm{c}$ ). The analogous statements for $S_{\lambda}$ are easily deduced from (2.35) and Theorem $2.18 b$ ). 
We thus have a real linear space $\operatorname{Sol}_{\lambda, C}$ endowed with a real-valued real bilinear non-degenerate symplectic form $\sigma_{\lambda}$. These data can now be used to proceed to a quantum field $\phi_{\lambda}$ satisfying the differential equation $D_{\lambda} \phi_{\lambda}=0$ by considering the corresponding CCR algebra $\mathfrak{A}_{\lambda}:=\operatorname{CCR}\left(\operatorname{Sol}_{\lambda, C}, \sigma_{\lambda}\right)$ over $\left(\operatorname{Sol}_{\lambda, C}, \sigma_{\lambda}\right)$ in a canonical manner [10].

On the level of the $C^{*}$-algebras $\mathfrak{A}_{\lambda}, \mathfrak{A}_{0}$, we have Bogoliubov isomorphisms $\alpha_{\lambda, \pm}: \mathfrak{A}_{\lambda} \rightarrow \mathfrak{A}_{0}$, induced by the Møller operators, and a scattering automorphism $s_{\lambda}: \mathfrak{A}_{0} \rightarrow \mathfrak{A}_{0}$, induced by $S_{\lambda}$.

The second case we want to consider is the more particular case of a Dirac operator $D$ as an example of a prenormally hyperbolic operator (with $D^{\prime}=-D$ ). Thus we take $D=-i \gamma^{\mu} \partial_{\mu}+V(x)$, where the $\gamma^{\mu}$ satisfy the Clifford relations [16], in particular, $\left(\gamma^{0}\right)^{*}=\gamma^{0}=\left(\gamma^{0}\right)^{-1}$ and $\left(\gamma^{k}\right)^{*}=-\gamma^{k}=\gamma^{0} \gamma^{k} \gamma^{0}, k=1, \ldots, s$. We restrict to dimension $n$ even or $n=3 \bmod 8$ or $n=9 \bmod 8$. Setting $N:=2^{n / 2}$ for $n$ even and $N:=2^{(n-1) / 2}$ for $n$ odd, one can then also find a charge conjugation for the Dirac matrices $\gamma^{\mu}$, that is an antiunitary involution $C: \mathbb{C}^{N} \rightarrow \mathbb{C}^{N}$ satisfying $C \gamma^{\mu} C=-\gamma^{\mu}, \mu=0,1, \ldots, s$. As before, we will use the same symbol $C$ to denote its pointwise action on functions taking values in $\mathbb{C}^{N}$.

We then have $C\left(-i \gamma^{\mu} \partial_{\mu}\right) C=-i \gamma^{\mu} \partial_{\mu}$, and upon requiring $C V(x) C=V(x)$, $C W C=W$, also $C D_{\lambda} C=D_{\lambda}$ (for $\lambda$ real). As before, this implies $C R_{\lambda}^{ \pm} C=R_{\lambda}^{ \pm}$.

Furthermore, the potential $V$ and the perturbation $W$ are required to satisfy $\gamma^{0} V \gamma^{0}=V^{*}, \gamma^{0} W \gamma^{0}=W^{*}$. In that case, we have $D_{\lambda}{ }^{*}=\gamma^{0} D_{\lambda} \gamma^{0}$, and thus $R_{\lambda}{ }^{*}=-\gamma^{0} R_{\lambda} \gamma^{0}$. Now we define

$$
\begin{aligned}
\delta_{\lambda}: \operatorname{Sol}_{\lambda} \times \operatorname{Sol}_{\lambda} & \rightarrow \mathbb{C}, \\
\delta_{\lambda}\left(R_{\lambda} f, R_{\lambda} g\right) & :=i \rho_{\lambda}\left(R_{\lambda} \gamma^{0} f, R_{\lambda} g\right)=i\left\langle f, \gamma^{0} R_{\lambda} g\right\rangle .
\end{aligned}
$$

\section{Proposition 4.2 (Hilbert space structure of Dirac field solution spaces).}

a) Under the assumptions made, $\left(\operatorname{Sol}_{\lambda}, \delta_{\lambda}\right)$ is a pre Hilbert space, with Hilbert space completion denoted $\mathcal{K}_{\lambda}$. If $\Sigma_{t}$ is a Cauchy hyperplane such that $K \subset \Sigma_{t}^{+}$or $K \subset \Sigma_{t}^{-}$, then

$$
\delta_{\lambda}\left(R_{\lambda} f, R_{\lambda} g\right)=\int_{\Sigma_{t}}\left(\left(R_{\lambda} f\right)_{t},\left(R_{\lambda} g\right)_{t}\right) .
$$

b) The Moller operators $\Omega_{\lambda, \pm}$ and the scattering operator $S_{\lambda}$ extend to unitaries $\Omega_{\lambda, \pm}: \mathcal{K}_{\lambda} \rightarrow \mathcal{K}_{0}$ and $S_{\lambda}: \mathcal{K}_{0} \rightarrow \mathcal{K}_{0}$, respectively.

c) The conjugation $C$ induces an antiunitary operator $C_{\lambda}$ on $\mathcal{K}_{\lambda}$ by $C_{\lambda} R_{\lambda} f:=$ $R_{\lambda} C f$. We have

$$
\Omega_{\lambda, \pm} C_{\lambda}=C_{0} \Omega_{\lambda, \pm}, \quad S_{\lambda} C_{0}=C_{0} S_{\lambda}
$$


Proof. a) As $R_{\lambda}{ }^{*}=-\gamma^{0} R_{\lambda} \gamma^{0}$, the mapping $\delta_{\lambda}\left(R_{\lambda} f, R_{\lambda} g\right)=-i\left\langle f, \gamma^{0} R_{\lambda} g\right\rangle=$ $i\left\langle R_{\lambda} f, \gamma^{0} g\right\rangle$ (4.4) is well-defined, and clearly sesquilinear.

Furthermore, once (4.5) is established, it is clear from this form that $\delta_{\lambda}$ is positive semidefinite. Actually, it is then definite: For if $\delta_{\lambda}\left(R_{\lambda} f, R_{\lambda} f\right)=0$, then the solution $R_{\lambda} f \in \operatorname{Sol}_{\lambda}$ vanishes on each Cauchy hyperplane $\Sigma_{t}$ such that $K \subset \Sigma_{t}$. Thus $R_{\lambda} f$ is supported in a light cone, which implies $R_{\lambda} f=0$.

So it remains to show (4.5). To this end, we proceed in an analogous fashion as in the proof of Proposition 2.12, see also [20, Prop. 1.2, Prop. 2.2], [13, Prop. 2.1], [4, Lemma 3.17], for similar arguments for Dirac operators without non-local perturbation.

Let $\Sigma_{t}$ be a Cauchy hyperplane such that $K \subset \Sigma_{t}^{+}$(the case $K \subset \Sigma_{t}^{-}$is analogous), $f, g \in \mathscr{C}_{0}^{\infty}$, and consider the vector fields

$$
X_{ \pm}^{\mu}(x):=-\left(\left(R_{\lambda}^{ \pm} f\right)(x), \gamma^{0} \gamma^{\mu}\left(R_{\lambda} g\right)(x)\right) .
$$

Using the relations of the Clifford algebra and $V(x)^{*}=\gamma^{0} V(x) \gamma^{0}$, we compute

$$
\begin{aligned}
& \partial_{\mu} X_{ \pm}^{\mu}(x)=-i\left(\left(R_{\lambda}^{ \pm} f\right)(x), \gamma^{0}\left(-i \gamma^{\mu} \partial_{\mu}\left(R_{\lambda} g\right)(x)\right)\right. \\
&+i\left(\left(-i \gamma^{\mu} \partial_{\mu}\left(R_{\lambda}^{ \pm} f\right)(x), \gamma^{0}\left(R_{\lambda} g\right)(x)\right)\right. \\
&=-i\left(\left(R_{\lambda}^{ \pm} f\right)(x), \gamma^{0}\left(D R_{\lambda} g\right)(x)\right)+i\left(\left(D R_{\lambda}^{ \pm} f\right)(x), \gamma^{0}\left(R_{\lambda} g\right)(x)\right) \\
&=i \lambda\left(\left(R_{\lambda}^{ \pm} f\right)(x), \gamma^{0}\left(W R_{\lambda} g\right)(x)\right)-i \lambda\left(\left(W R_{\lambda}^{ \pm} f\right)(x), \gamma^{0}\left(R_{\lambda} g\right)(x)\right) \\
&+i\left(f(x), \gamma^{0}\left(R_{\lambda} g\right)(x)\right) .
\end{aligned}
$$

The support of $X_{+}^{\mu}$ has compact intersection with $\Sigma_{t}^{\mp}$. Making use of Gauss' theorem, $\pm \int_{\Sigma_{t}}\left(X_{ \pm}^{ \pm}\right)_{t}=\int_{\Sigma_{t} \mp} \partial_{\mu} X_{ \pm}^{\mu}$, and taking into account $\left(\gamma^{0}\right)^{2}=1$, we get

$$
\begin{aligned}
\mp \int_{\Sigma_{t}}\left(\left(R_{\lambda}^{ \pm} f\right)_{t},\left(R_{\lambda} g\right)_{t}\right)= & i \int_{\Sigma_{t}^{ \pm}}\left(f, \gamma^{0} R_{\lambda} g\right)+i \lambda \int_{\Sigma_{t}^{ \pm}}\left(R_{\lambda}^{ \pm} f, \gamma^{0} W R_{\lambda} g\right) \\
& -i \lambda \int_{\Sigma_{t}^{ \pm}}\left(W R_{\lambda}^{ \pm} f, \gamma^{0} R_{\lambda} g\right) \\
= & i \int_{\Sigma_{t}^{ \pm}}\left(f, \gamma^{0} R_{\lambda} g\right),
\end{aligned}
$$

where in the second step, we have used $K \subset \Sigma^{+}$and $\gamma^{0} W \gamma^{0}=W^{*}$.

Adding the equations for both choices of " \pm " gives the claimed equation (4.5):

$$
\int_{\Sigma_{t}}\left(\left(R_{\lambda} f\right)_{t},\left(R_{\lambda} g\right)_{t}\right)=i\left\langle f, \gamma^{0} R_{\lambda} g\right\rangle=\delta_{\lambda}\left(R_{\lambda} f, R_{\lambda} g\right) .
$$

b) Let $f^{ \pm} \in \mathscr{C}_{0}^{\infty}\left(\Sigma_{\tau^{ \pm}}^{ \pm}\right)$. Then $\Omega_{\lambda, \pm} R_{\lambda} f^{ \pm}=R f^{ \pm}$, and with Proposition $2.17 c$ ), we get

$$
\begin{aligned}
\delta_{0}\left(\Omega_{\lambda, \pm} R_{\lambda} f^{ \pm}, \Omega_{\lambda, \pm} R_{\lambda} g^{ \pm}\right) & =\delta_{0}\left(R f^{ \pm}, R g^{ \pm}\right)=i \rho_{0}\left(R \gamma^{0} f^{ \pm}, R g^{ \pm}\right) \\
& =i \rho_{\lambda}\left(R_{\lambda} \gamma^{0} f^{ \pm}, R_{\lambda} g^{ \pm}\right)=\delta_{\lambda}\left(R_{\lambda} f^{ \pm}, R_{\lambda} g^{ \pm}\right) .
\end{aligned}
$$


As the spaces $\left.R_{\lambda} \mathscr{C}_{0}^{\infty} \Sigma_{\tau^{ \pm}}^{ \pm}\right) \subset \mathcal{K}_{\lambda}$ and $R_{0} \mathscr{C}_{0}^{\infty}\left(\Sigma_{\tau^{ \pm}}^{ \pm}\right) \subset \mathcal{K}_{0}$ are dense by construction of $\mathcal{K}_{\lambda}, \mathcal{K}_{0}$, this shows that $\Omega_{\lambda, \pm}$ are linear isometries with dense domains and ranges, and therefore extend to unitaries. The scattering operator is also unitary as the product of $\Omega_{\lambda,+}$ and $\Omega_{\lambda,-}{ }^{-1}$.

c) As $R_{\lambda}$ and $C$ commute, $C_{\lambda}$ is well defined, and in view of

$$
\begin{aligned}
\delta_{\lambda}\left(C_{\lambda} R_{\lambda} f, C_{\lambda} R_{\lambda} g\right) & =i\left\langle C f, \gamma^{0} R_{\lambda} C g\right\rangle \\
& =\overline{-i\left\langle f, C \gamma^{0} C R_{\lambda} g\right\rangle}=\overline{i\left\langle f, \gamma^{0} R_{\lambda} g\right\rangle}=\overline{\delta_{\lambda}\left(R_{\lambda} f, R_{\lambda} g\right)},
\end{aligned}
$$

also antiunitary. The commutation relations (4.6) follow directly from the definition of the Møller operators and $C R_{\lambda} C=R_{\lambda}$.

In the case of a perturbed Dirac operator, we have thus constructed a family of Hilbert spaces $\mathcal{K}_{\lambda}$ with antiunitary involutions $C_{\lambda}$, and can use these data to proceed to the CAR algebras $\mathfrak{F}_{\lambda}:=\operatorname{CAR}\left(\mathcal{K}_{\lambda}, C_{\lambda}\right)$ over $\left(\mathcal{K}_{\lambda}, C_{\lambda}\right)$ [10], similarly to the CCR case for symmetric $D_{\lambda}$. Again we have induced Bogoliubov isomorphisms $\alpha_{\lambda, \pm}: \mathfrak{F}_{\lambda} \rightarrow \mathfrak{F}_{0}$ on the level of the $C^{*}$-algebras $\mathfrak{F}_{\lambda}$ and $\mathfrak{F}_{0}$, induced by the Møller operators, and a scattering automorphism $s_{\lambda}: \mathfrak{F}_{0} \rightarrow \mathfrak{F}_{0}$, induced by $S_{\lambda}$. These structures form the prerequisites for a systematic study of the corresponding quantum Dirac fields.

\section{Outlook}

We would like to see the problem investigated in this work as one step towards finding an answer to the question of how to characterize the basic structural properties of quantum fields on noncommutative geometries in a model-independent manner. Here, model-independent not only refers to quantum field theory but also to noncommutative geometry. While there is a powerful model-independent framework that allows it to discuss and analyse structural properties of quantum field theories on classical ("commutative") spacetimes [30], it is not so clear if there is a comparably developed model-independent conceptual and mathematical framework within which to stage a discussion of quantum field theories on noncommutative geometries, or even of noncommutative geometries as such. However, one may - at least as a working hypothesis - take the point of view that Connes' spectral geometry approch to noncommutative geometry [15] subsumes most relevant noncommutative geometries, and that this even holds true for the case of noncommutative spacetimes with Lorentzian signature upon suitably generalizing the spectral geometry framework [24, 25, 36, 47].

In fact, Moyal-deformed Minkowski spacetime appears to fit in with a suitably generalized spectral geometry set-up; yet for the locally deformed products discussed above this is not at all clear and remains at present an open question. 
Nonetheless, it seems possible that a (generalized) spectral triple can be associated with Moyal-Minkowski spacetime, having as main ingredients a Hilbert space $\mathcal{H}$, a *-algebra of bounded operators $\mathcal{A}$ on $\mathcal{H}$ and a Dirac operator $D$. Further elements of structure include a time-orientation operator (encoding also Lorentzian metric signature - and which scalar product on the space of spinor fields has been selected) and a charge conjugation, as well as additional pieces of data; we refrain from entering into details of these matters (which do not seem to have reached a final form at the present time) and refer to $[13,48]$ for fuller discussion. For the case of MoyalMinkowski spacetime, $\mathcal{H}$ corresponds to an $L^{2}$-space of spinor fields on Minkowski spacetime, $D$ is the usual Dirac operator on Minkowski spacetime, and $\mathcal{A}$ may be taken as the Schwartz functions on Minkowski spacetime endowed with the Moyal product as algebra product. In contrast, the spectral triple of ordinary "commutative" Minkowski spacetime uses the same $\mathcal{H}$ and $D$, but takes the Schwartz functions with the usual pointwise product of functions as the algebra $\mathcal{A}$.

To explain how the results of the present article can be used to identify relevant quantum observables in a canonical quantum field theory associated with these geometric data, we first consider the case of "commutative" Minkowski spacetime. Here the quantized Dirac field can be viewed as arising from a form of second quantization of the spectral triple associated to Minkowski spacetime. One introduces a suitable smooth domain, $\mathcal{H}_{D}$, of $D$ in $\mathcal{H}$; a choice may be $\mathscr{C}_{0}^{\infty}$. Second quantization renders a CAR algebra $\mathfrak{F}$ fixed by $\mathcal{H}_{D}$ and $D$, as follows: $\mathfrak{F}$ is the unique unital $C^{*}$-algebra generated by elements $\psi(f), f \in \mathcal{H}_{D}$, which satisfy $\psi(D f)=0$ and

$$
\psi\left(f_{1}\right)^{*} \psi\left(f_{2}\right)+\psi\left(f_{2}\right) \psi\left(f_{1}\right)^{*}=i\left(f_{1}, R f_{2}\right) \cdot 1 .
$$

where, as before, $R=R^{-}-R^{+}$denotes the difference of advanced and retarded fundamental solutions to the Dirac operator $D$, and $(\cdot, \cdot)$ is the scalar product of $\mathcal{H}$. In the notation of the previous section, we thus have $\left(f_{1}, R f_{2}\right)=i\left\langle f_{1}, \gamma^{0} R f_{2}\right\rangle$, and $\mathfrak{F}$ coincides with the $C^{*}$-algebra $\mathfrak{F}_{0}$ constructed there.

The generating elements $\psi(f), f \in \mathcal{H}_{D}$, of $\mathfrak{F}$ can be seen as abstract quantized Dirac field operators. Observable quantum fields associated with some $w \in C_{0}^{\infty}$ can be obtained via scattering processes, viewing $w$ as a potential. In fact, when passing from $D$ to $D+\lambda W$, where $W$ acts by pointwise multiplication with $w$, we are in the situation considered in Sections 2.3 and 4, and obtain scattering morphisms $s_{\lambda}$ on $\mathfrak{F}$. In the spirit of "Bogoliubov's formula" [11], the derivative of these morphisms w.r.t. the coupling yields [13]

$$
\left.\frac{d}{d \lambda} s_{\lambda}(\psi(f))\right|_{\lambda=0}=\psi(w \cdot R f)=i\left[: \psi^{+} \psi:(w), \psi(f)\right],
$$

where on the right hand side, $: \psi^{+} \psi:(w)$ is the Wick-product (in vacuum representation) of the adjoint quantized Dirac field and the quantized Dirac field 
- which is a scalar quantum field, hence observable, with the interpretation of a quantized field density.

We propose to use the exactly same approach to obtain also the relevant observables for a quantum field theory over a noncommutative spectral geometry, and explain this here at the example of noncommutative Minkowski space. Replacing in the above example the algebra $\mathcal{A}$ by Schwartz functions $\mathcal{A}_{\star}$, but equipped with the Moyal-Weyl product, passing to a second quantized Dirac quantum field theory yields the identical $C^{*}$-algebra $\mathfrak{F}=\mathfrak{F}_{0}$; simply because the product in $\mathcal{A}_{\star}$ is not used in the construction. To see where information about non-commutativity of the underlying spacetime may be stored in the second quantized quantum field theory, one may again look at scattering transformations mediated by elements of the algebra $\mathcal{A}_{\star}$ in the spectral geometry data. Fixing some $w \in C_{0}^{\infty}$ as before, we thus again pass from the Dirac operator $D$ to $D+\lambda W_{\star}$, where now $W_{\star}$ acts by Moyal-multiplication with $w$, i.e. $W_{\star} f=w \star f$. This perturbation is the limit as $\varepsilon \rightarrow 0$ of a sequence of $C_{0}^{\infty}$-kernel operators $W_{\star, \varepsilon}$, to which the results of the present article apply (see Section 3).

In the case of a Moyal product with commutative time (where a Hamiltonian formulation of the differential equation is at hand), it was shown in [13] that the corresponding scattering morphism $s_{\lambda}$ takes the form

$$
\left.\lim _{\varepsilon \rightarrow 0} \frac{d}{d \lambda} s_{\varepsilon, \lambda}(\psi(f))\right|_{\lambda=0}=\psi(w \star R f)=i\left[: \psi^{+} \psi:(w)^{\star}, \psi(f)\right],
$$

where on the right hand side, there is a suitably Moyal-Rieffel deformed version of the commutator. More interesting, however, is the fact that one can expect that there are (symmetric) operators $X(w)$ such that

$$
\left.\lim _{\varepsilon \rightarrow 0} \frac{d}{d \lambda} s_{\varepsilon, \lambda}(\psi(f))\right|_{\lambda=0}=i[X(w), \psi(f)]
$$

and these operators would obviously be different from the Wick products : $\psi^{+} \psi:(w)$. In particular, they have completely different localization properties. In the spirit of Bogoliubov's formula, the operators $X(w)$ should be regarded as "observables" of the quantum field associated with $w$ regarded as an element of the MoyalRieffel deformed "test-functions" over Minkowski spacetime, i.e. the algebra $\mathcal{A}_{\star}$ of the spectral geometry data of Moyal-Minkowski spacetime, see [13, 48] for further discussion. Following this line of argument offers a systematic way to associate quantum field observables to elements of an "algebra of non-commutative coordinates" seen, at least tentatively, from a perspective of Connes' spectral geometry. The relation (5.1) suggests that there should be a close relation between the algebra generated by the $X(w)$ and warped convolutions of Dirac quantum field operators as investigated in [7]. We hope to gain further insight about such a possible relationship elsewhere. 
Acknowledgements. We thank Alexander Strohmaier for his comment on the analytic Fredholm theorem.

\section{References}

[1] N. Afshordi, S. Aslanbeigi, R. Sorkin. A distinguished vacuum state for a quantum field in a curved spacetime: formalism, features, and cosmology. $J$. High Energy Phys. 2012 (2012), no. 8, 137. MR 3006915

[2] M. Benini, C. Dappiaggi and T.-P. Hack. Quantum field theory on curved backgrounds - A primer. Int. Jour. Mod. Phys. A 28 (2013), no. 17, 1330023. arXiv:1306.0527 Zbl 1272.81128 MR 3081406

[3] R. Brunetti, K. Fredenhagen and R. Verch. The generally covariant locality principle - A new paradigm for local quantum field theory. Commun. Math. Phys. 237 (2003), no. 1-2, 31-68. arXiv:math-ph/0112041 Zbl 1047.81052 MR 2007173

[4] C. Bär and N. Ginoux. Classical and Quantum Fields on Lorentzian Manifolds in Global Differential Geometry, 359-400, Springer Proc. Math., 17, Springer, Heidelberg, 2012. arXiv:1104.1158 Zbl 1254.81044 MR 3289848

[5] C. Bär, N. Ginoux and F. Pfäffle. Wave Equations on Lorentzian Manifolds and Quantization. ESI Lectures in Mathematics and Physics. European Mathematical Society (EMS), Zürich, 2007. Zbl 1118.58016 MR 2298021

[6] N. Bogolubov, A. A. Logunov, A. I. Oksak and I. T. Todorov. General principles of quantum field theory. Kluwer Academic Publishers Group, Dordrecht, 1990. Zbl 0732.46040 MR 1135574

[7] D. Buchholz, G. Lechner and S. J. Summers. Warped convolutions, Rieffel deformations and the construction of quantum field theories. Commun. Math. Phys. 304 (2011), no. 1, 95-123. arXiv:1005.2656 Zbl 1227.46043 MR 2793931

[8] M. Borris. Quantum Field Theory on Non-commutative Spacetimes. Ph. D. thesis, Leipzig (2011). http://www.qucosa.de/recherche/frontdoor/ ?tx_slubopus4frontend [id] $=6778$

[9] O. Bratteli and D. W. Robinson. Operator Algebras and Quantum Statistical Mechanics I. $C^{*}$ - and $W^{*}$-algebras, symmetry groups, decomposition of states. Second edition. Texts and Monographs in Physics. Springer-Verlag, New York, 1987. Zbl 0905.46046 MR 887100

[10] O. Bratteli and D. W. Robinson. Operator algebras and quantum-statistical mechanics. II. Equilibrium states. Models in quantum-statistical mechanics. Texts and Monographs in Physics. Springer-Verlag, New York-Berlin, 1997. Zbl 0463.46052 MR 611508 
[11] N. N. Bogolubov and D. V. Shirkov. Introduction to the Theory of Quantized Fields. John Wiley \& Sons, New York-Chichester-Brisbane, 1980. Zbl 0925.81001 MR 579493

[12] D. Buchholz and S. J. Summers. Warped convolutions: A novel tool in the construction of quantum field theories in E. Seiler and K. Sibold (eds.), Quantum field theory and beyond: Essays in honor of Wolfhart Zimmermann, 107-121. World Sci. Publ., Hackensack, NJ, 2008. arXiv:0806.0349 Zbl 1206.81072 MR 2588578

[13] M. Borris and R. Verch. Dirac field on Moyal-Minkowski spacetime and noncommutative potential scattering. Commun. Math. Phys. 293 (2010), 399-448. arXiv:0812.0786 Zbl 1208.81189 MR 2563789

[14] D. Bahns and S. Waldmann. Locally noncommutative space-times. Rev. Math. Phys. 19 (2007), 273-306. arXiv:math/0607745 Zbl 1127.81027 MR 2316535

[15] A. Connes. Noncommutative Geometry. InterEditions, Paris, 1990. Zbl 0745.46067 MR 1079062

[16] R. Coquereaux. Spinors, Reflections, and Clifford Algebras: A Review. In Spinors in Physics and Geometry (Trieste, 1986), 135-190, World Sci. Publ., Singapore, 1988. Zbl 0947.53501 MR 992414

[17] S. Doplicher, K. Fredenhagen and J. E. Roberts. The Quantum structure of space-time at the Planck scale and quantum fields. Commun. Math. Phys. 172 (1995), 187-220. arXiv:hep-th/0303037 Zbl 0847.53051 MR 1346376

[18] B. Durhuus and V. Gayral. The Scattering problem for a noncommutative nonlinear Schrödinger equation. SIGMA 6 (2010), 046. Zbl 1219.35278 MR 2725037

[19] J. Duistermaat and L. Hörmander. Fourier Integral Operators II. Acta Math. 128 (1972), 183-269. Zbl 0232.47055 MR 388464

[20] J. Dimock. Dirac quantum fields on a manifold. Trans. Amer. Math. Soc. 269 (1982), 133-147. Zbl 0518.58018 MR 637032

[21] M. R. Douglas and N. A. Nekrasov. Noncommutative field theory. Rev. Mod. Phys. 73 (2001), 977-1029. arXiv:hep-th/0106048 Zbl 1205.81126 MR 1878801

[22] C. J. Fewster and R. Verch. Dynamical locality and covariance: What makes a physical theory the same in all spacetimes? Ann. Henri Poincaré 13 (2012), 1613-1674. arXiv:1106.4785 Zbl 1280.81099 MR 2982635

[23] C. J. Fewster and R. Verch. On a recent construction of 'vacuum-like' quantum field states in curved spacetime. Class. Quant. Grav. 29 (2012), 205017. Zbl 1256.83013 MR 2978968 
[24] N. Franco. Temporal Lorentzian spectral triples. Rev. Math. Phys. 26 (2014), 1430007. arXiv:1210.6575 Zbl 1305.58007 MR 3256858

[25] N. Franco and M. Eckstein. Noncommutative geometry, Lorentzian structures and causality in M. Eckstein, M. Heller, S.J. Szybka (eds.), Mathematical Structures of the Universe, CCPress, 2014. arXiv:1409.1480

[26] V. Gayral, J. M. Gracia-Bondia, B. Iochum, T. Schucker and J. C. Varilly. Moyal planes are spectral triples. Commun. Math. Phys. 246 (2004), 569-623. arXiv:hep-th/0307241 Zbl 1084.58008 MR 2053945

[27] H. Grosse and G. Lechner. Wedge-local quantum fields and noncommutative Minkowski space. J. High Energy Phys. 2007 (2007), no. 11, 012. arXiv:0706.3992 Zbl 1245.81048 MR 2362137

[28] H. Grosse and G. Lechner. Noncommutative deformations of Wightman quantum field theories. J. High Energy Phys. 2008 (2008), no. 09, 131. arXiv:0808.3459 Zbl 1245.81069 MR 2447659

[29] H. Grosse and R. Wulkenhaar. Renormalisation of $\phi^{4}$-theory on noncommutative $\mathbb{R}^{4}$ in the matrix base. Commun. Math. Phys. 256 (2005), 305-374. arXiv:hep-th/0401128 Zbl 1115.81055 MR 2160797

[30] R. Haag. Local Quantum Physics - Fields, Particles, Algebras, second edition. Texts and Monographs in Physics. Springer-Verlag, Berlin, 1996. Zbl 0857.46057 MR 1405610

[31] J. G. Heller, N. Neumaier and S. Waldmann. A C*-algebraic model for locally noncommutative spacetimes. Lett. Math. Phys. 80 (2007), 257-272. arXiv:math/0609850 Zbl 1127.53075 MR 2333512

[32] S. Hollands and R. M. Wald. Local Wick polynomials and time ordered products of quantum fields in curved spacetime. Commun. Math. Phys. 223 (2001), 289-326. arXiv:gr-qc/0103074 Zbl 0989.81081 MR 1864435

[33] S. Hollands and R. M. Wald. Axiomatic quantum field theory in curved spacetime. Commun. Math. Phys. 293 (2010), 85-125. arXiv:0803.2003 Zbl 1193.81076 MR 2563799

[34] G. Lechner and S. Waldmann. Strict deformation quantization of locally convex algebras and modules. Preprint, to appear in J. Geom. Phys., 2011. arXiv: 1109.5950

[35] R. Mühlhoff. Cauchy problem and Green's functions for first order differential operators and algebraic quantization. J. Math. Phys. 52 (2011), 022303. arXiv:1001.4091 MR 2798385

[36] M. Paschke and A. Sitarz. Equivariant Lorentzian spectral triples. Preprint, 2006. arXiv:math-ph/0611029 
[37] M. A. Rieffel. Deformation Quantization for Actions of $\mathbf{R}^{d}$, Mem. Amer. Math. Soc. 106 (1993), no. 506. Zbl 0798.46053 MR 1184061

[38] V. Rivasseau. Non-commutative renormalization in Séminaire Poincaré X, 1595, 2007. arXiv:0705.0705

[39] M. Reed and B. Simon. Methods of Modern Mathematical Physics I. Functional Analysis, Academic Press, New York-London, 1972. Zbl 0459.46001 MR 493419

[40] K. Sanders. The locally covariant Dirac field. Rev. Math. Phys. 22 (2010), 381430. arXiv:0911.1304 Zbl 1216.81113 MR 2647041

[41] M. A. Soloviev. On the failure of microcausality in noncommutative field theories. Phys. Rev. D77 (2008), no. 12, 125013. arXiv:0802.0997 MR 2434811

[42] R. F. Streater and A. Wightman. PCT, Spin and Statistics, and All That. Benjamin-Cummings, Reading, MA,1964. Zbl 0135.44305 MR 161603

[43] G. R. Sell and Y. You. Dynamics of Evolutionary Equations, Applied Mathematical Sciences, 143. Springer-Verlag, New York, 2002. Zbl 1254.37002 MR 1873467

[44] R. J. Szabo. Quantum field theory on noncommutative spaces. Phys. Rept. 378 (2003), 207-299. arXiv:hep-th/0109162 Zbl 1042.81581 MR 1971136

[45] B. Thaller. The Dirac Equation. Texts and Monographs in Physics. SpringerVerlag, Berlin, 1991. Zbl 0765.47023 MR 1219537

[46] F. Trèves. Topological vector spaces, distributions, and kernels. Academic Press, New York-London, 1967. Zbl 0171.10402 MR 225131

[47] K. van den Dungen, M. Paschke and A. Rennie. Pseudo-Riemannian spectral triples and the harmonic oscillator. J. Geom. Phys. 73 (2013), 37-55. arXiv:1207.2112 Zbl 1285.53060 MR 3090101

[48] R. Verch. Quantum Dirac field on Moyal-Minkowski spacetime - Illustrating quantum field theory over Lorentzian spectral geometry. Acta Phys. Pol. B Proc. Suppl. 4 (2011), 267-292. arXiv:1106.1138

[49] R. M. Wald. Quantum field theory in curved space-time and black hole thermodynamics. Chicago Lectures in Physics. University of Chicago Press, Chicago, IL, 1994. Zbl 0842.53052 MR 1302174

[50] S. Waldmann. Geometric Wave Equations. Lecture Notes, 2012. arXiv:1208.4706 
Received 12 December, 2013; revised 03 November, 2014

G. Lechner, Institut für Theoretische Physik, Universität Leipzig, Brüderstr. 16, 04103 Leipzig, Germany

Present address: School of Mathematics, Cardiff University, Senghennydd Road, Cardiff CF24 4AG, UK

E-mail: LechnerG@ cardiff.ac.uk

R. Verch, Institut für Theoretische Physik, Universität Leipzig, Brüderstr. 16,

04103 Leipzig, Germany

E-mail: rainer.verch@uni-leipzig.de 